Pacific

Journal of

Mathematics

\title{
ENDOSCOPIC TRANSFER FOR UNITARY GROUPS AND HOLOMORPHY OF ASAI $L$-FUNCTIONS
}

Neven Grbac and Freydoon Shahidi 


\title{
ENDOSCOPIC TRANSFER FOR UNITARY GROUPS AND HOLOMORPHY OF ASAI L-FUNCTIONS
}

\author{
Neven Grbac AND Freydoon Shahidi
}

\begin{abstract}
The analytic properties of the complete Asai $L$-functions attached to cuspidal automorphic representations of the general linear group over a quadratic extension of a number field are obtained. The proof is based on the comparison of the Langlands-Shahidi method and Mok's endoscopic classification of automorphic representations of quasisplit unitary groups.
\end{abstract}

$\begin{array}{lr}\text { Introduction } & 185 \\ \text { 1. The quasisplit unitary groups } & 187 \\ \text { 2. Eisenstein series and Asai } L \text {-functions } & 189 \\ \text { 3. Arthur parameters for unitary groups } & 198 \\ \text { 4. Holomorphy and nonvanishing of Asai } L \text {-functions } & 203 \\ \text { Acknowledgements } & 208 \\ \text { References } & 208\end{array}$

\section{Introduction}

In this paper we study the analytic properties of the complete Asai $L$-function attached to a cuspidal automorphic representation of the general linear group over a quadratic extension of a number field. The approach is based on the LanglandsShahidi method, combined with the knowledge of the poles of Eisenstein series coming from a recent endoscopic classification of automorphic representations of the quasisplit unitary groups by Mok [2015].

In order to state the main result more precisely, we introduce some notation. Let $E / F$ be a quadratic extension of number fields, and let $\theta$ be the unique nontrivial element in the Galois group $\operatorname{Gal}(E / F)$. Let $\mathbb{A}_{E}$ and $\mathbb{A}_{F}$ be the rings of adèles of $E$ and $F$, respectively. Let $\hat{\delta}$ be any extension to $A_{E}^{\times} / E^{\times}$of the quadratic character of $\mathbb{A}_{F}^{\times} / F^{\times}$attached to $E / F$ by class field theory.

Neven Grbac is supported in part by Croatian Science Foundation under the project 9364 and by University of Rijeka research grant 13.14.1.2.02. Freydoon Shahidi is partially supported by NSF grant DMS1162299.

MSC2010: 11F66, 11F70, 11F72, 22E55.

Keywords: Asai $L$-function, automorphic representation, endoscopic classification, spectral decomposition, quasisplit unitary group. 
For a cuspidal automorphic representation $\sigma$ of $\mathrm{GL}_{n}\left(\mathbb{A}_{E}\right)$, we denote by $\sigma^{\theta}$ its Galois conjugate and by $\tilde{\sigma}$ its contragredient representation. We say that $\sigma$ is Galois self-dual if $\sigma \cong \tilde{\sigma}^{\theta}$, that is, $\sigma$ is isomorphic to its Galois conjugate contragredient.

Let $L\left(s, \sigma, r_{A}\right)$ denote the complete Asai $L$-function attached to $\sigma$ and the Asai representation $r_{A}$ via the Langlands-Shahidi method. See Section 2.A for a definition. Our main result on the holomorphy and nonvanishing of the Asai $L$-function $L\left(s, \sigma, r_{A}\right)$ is the following theorem.

Theorem 4.3. Let $\sigma$ be a cuspidal automorphic representation of $\mathrm{GL}_{n}\left(\mathbb{A}_{E}\right)$. Let $L\left(s, \sigma, r_{A}\right)$ (respectively, $\left.L\left(s, \sigma \otimes \hat{\delta}, r_{A}\right)\right)$ be the Asai (respectively, twisted Asai) $L$-function attached to $\sigma$, where $\hat{\delta}$ is any extension to $\mathbb{A}_{E}^{\times} / E^{\times}$of the quadratic character of $\mathbb{A}_{F}^{\times} / F^{\times}$attached to the extension $E / F$ by class field theory.

(1) If $\sigma$ is not Galois self-dual, i.e., if $\sigma \not \tilde{\sigma}^{\theta}$, then $L\left(s, \sigma, r_{A}\right)$ is entire. It is nonzero for $\operatorname{Re}(s) \geq 1$ and $\operatorname{Re}(s) \leq 0$.

(2) If $\sigma$ is Galois self-dual, i.e., if $\sigma \cong \tilde{\sigma}^{\theta}$, then

(a) $L\left(s, \sigma, r_{A}\right)$ is entire, except for possible simple poles at $s=0$ and $s=1$, and nonzero for $\operatorname{Re}(s) \geq 1$ and $\operatorname{Re}(s) \leq 0$;

(b) exactly one of the L-functions $L\left(s, \sigma, r_{A}\right)$ and $L\left(s, \sigma \otimes \hat{\delta}, r_{A}\right)$ has simple poles at $s=0$ and $s=1$, while the other is holomorphic at those points.

The idea of the proof is to consider the Eisenstein series attached to $\sigma$ on the quasisplit unitary group $U_{2 n}\left(\mathbb{A}_{F}\right)$ defined by the quadratic extension $E / F$, where $\sigma$ is viewed as a representation of the Levi factor of the Siegel maximal parabolic subgroup of $U_{2 n}$ in $2 n$ variables. We look at the contribution of this Eisenstein series to the residual spectrum from two different points of view. On the one hand, by the Langlands-Shahidi method [2010], the poles of the Eisenstein series for the complex argument in the positive Weyl chamber are determined by certain ratio of the Asai $L$-functions. The residues at such a pole span a residual representation of $U_{2 n}\left(\mathbb{A}_{F}\right)$. On the other hand, this residual representation should have an Arthur parameter, according to Mok's endoscopic classification [2015] of automorphic representations of quasisplit unitary groups (see also [Arthur 2005; 2013]). Comparing the possible Arthur parameters and poles of Asai $L$-functions, we are able to deduce the analytic properties of these $L$-functions.

Mok's work, as well as Arthur's, still depends on the stabilization of the twisted trace formula for the general linear group. Hence, our result is also conditional on this stabilization. This issue is considered by Waldspurger [2014a; 2014b; 2014c]. In our paper, we always make a remark when a partial result could have been obtained without using Mok's work. In fact, the crucial insight coming from endoscopic classification is holomorphy of the Asai $L$-function $L\left(s, \sigma, r_{A}\right)$ inside the critical strip $0<\operatorname{Re}(s)<1$. 
This method was applied in [Grbac 2011] to the complete exterior and symmetric square $L$-functions attached to a cuspidal automorphic representation of $\mathrm{GL}_{n}\left(\mathbb{A}_{F}\right)$. It relies on Arthur's endoscopic classification of automorphic representations for split classical groups [Arthur 2013; 2005]. The result and the approach are of the same nature as the above theorem for Asai $L$-functions. The approach has also already been used as a part of a long term project to study endoscopy via descent by Jiang, Liu and Zhang [Jiang et al. 2013].

A different approach to describing the analytic properties of $L$-functions is that of integral representations. However, this approach usually gives holomorphy of partial $L$-functions, which is weaker than our result due to ramification or problems at archimedean places. For the exterior square $L$-function this was pursued in [Bump and Friedberg 1990; Kewat and Raghunathan 2012; Belt 2012], for the symmetric square $L$-function in [Bump and Ginzburg 1992] and more generally for twisted symmetric square in a series of papers [Takeda 2014b; 2014a; 2013], and for the Asai $L$-functions in [Flicker 1988; Flicker and Zinoviev 1995; Anandavardhanan and Rajan 2005].

The paper is organized as follows. In Section 1 we introduce the unitary group structure and fix the notation. In Section 2 the relation between poles of Eisenstein series on quasisplit unitary groups and the Asai $L$-functions is investigated. Section 3 provides a definition of Arthur parameters and packets for quasisplit unitary groups in terms of results of Mok. Finally, in Section 4 we prove the main result on the analytic properties of Asai $L$-functions.

\section{The quasisplit unitary groups}

1.A. Definition and basic structure. Let $E / F$ be a quadratic extension of number fields. The nontrivial Galois automorphism in the Galois group $\operatorname{Gal}(E / F)$ is denoted by $\theta$. Let $N_{E / F}$ denote the norm map from $E$ to $F$. Let $\mathbb{A}_{F}$ and $\mathbb{A}_{E}$ be the rings of adèles of $F$ and $E$, respectively, and $\mathbb{A}_{F}^{\times}$and $\mathbb{A}_{E}^{\times}$the corresponding groups of idèles.

The quadratic character of $\mathbb{A}_{F}^{\times} / F^{\times}$attached to $E / F$ by class field theory is denoted by $\delta_{E / F}$. We always identify $\delta_{E / F}$ with the corresponding character of the Weil group $W_{F}$ of $F$ under class field theory. Let $\hat{\delta}$ be any extension of $\delta_{E / F}$ to a character of $\mathbb{A}_{E}^{\times} / E^{\times}$. Such extension is not unique.

We denote by $F_{v}$ the completion of $F$ at the place $v$. If a place $v$ of $F$ does not split in $E$, we always denote by $w$ the unique place of $E$ lying over $v$. Then $E_{w} / F_{v}$ is a quadratic extension of local fields. If $v$ splits in $E$, we denote by $w_{1}$ and $w_{2}$ the two places of $E$ lying over $v$. Then we have $E_{w_{1}} \cong E_{w_{2}} \cong F_{v}$. We use $F_{\infty}$ to denote the product of $F_{v}$ over archimedean places.

For an integer $N \geq 2$, we consider in this paper the $F$-quasisplit unitary group $U_{N}$ in $N$ variables defined by the extension $E / F$, viewed as an algebraic group 
over $F$. More precisely, $U_{N}$ is a group scheme over $F$, whose functor of points is defined as follows. Consider $\theta$ as an element of the Galois group $\operatorname{Gal}(\bar{F} / F)$ trivial on $\bar{F} / E$, where $\bar{F}$ is a fixed algebraic closure of $F$. Let $V$ be an $N$-dimensional vector space over $E$. We fix a form on $V$ as in [Kim and Krishnamurthy 2004; 2005], that is, let

$$
J_{n}=\left(.{ }^{1}\right) \quad \text { and } \quad J_{N}^{\prime}= \begin{cases}\left(\begin{array}{cc}
0 & J_{n} \\
-J_{n} & 0
\end{array}\right) & \text { for } N=2 n \\
\left(\begin{array}{ccc}
0 & 0 & J_{n} \\
0 & 1 & 0 \\
-J_{n} & 0 & 0
\end{array}\right) & \text { for } N=2 n+1 .\end{cases}
$$

Then the functor of points of $U_{N}$ is given by

$$
U_{N}(R)=\left\{g \in \mathrm{GL}_{E \otimes_{F} R}\left(V \otimes_{F} R\right):{ }^{*} g J_{N}^{\prime} g=J_{N}^{\prime}\right\}
$$

for any $F$-algebra $R$, where ${ }^{*} g={ }^{t} g^{\theta}$ is the conjugate transpose of $g$. In particular, the $F$-points of $U_{N}$ are given as

$$
U_{N}(F)=\left\{g \in \mathrm{GL}_{N}(E):{ }^{*} g J_{N}^{\prime} g=J_{N}^{\prime}\right\} .
$$

Writing $N=2 n$ if $N$ is even, and $N=2 n+1$ if $N$ is odd, the $F$-rank of $U_{N}$ in both cases equals $n \geq 1$. For $N=1$, the unitary group $U_{1}$ in one variable is obtained by inserting $N=1$ in the definition of $U_{N}$. Its $F$-points are nothing else than

$$
U_{1}(F)=\left\{x \in E^{\times}: \theta(x) x=1\right\},
$$

which is the norm-one subgroup $E^{1}$ of $E^{\times}$.

For $m \geq 1$, let $G_{m}=\operatorname{Res}_{E / F} \mathrm{GL}_{m}$ be the algebraic group over $F$ obtained from the general linear group $\mathrm{GL}_{m}$ over $E$ by restriction of scalars from $E$ to $F$. If $m \leq n$, it appears in the Levi factors of parabolic subgroups of $U_{N}$.

We fix the Borel subgroup $P_{0}$ of $U_{N}$ consisting of upper-triangular matrices. Let $P_{0}=M_{0} N_{0}$, where $M_{0}$ is a maximally split maximal torus of $U_{N}$ (i.e., one containing a maximal split torus of $U_{N}$; see [Shahidi 2010, Chapter I]), and $N_{0}$ the unipotent radical of $P_{0}$. Then

$$
M_{0} \cong \begin{cases}G_{1} \times \cdots \times G_{1} & \text { for } N=2 n, \\ G_{1} \times \cdots \times G_{1} \times U_{1} & \text { for } N=2 n+1,\end{cases}
$$

with $n$ copies of $G_{1}$, so that the $F$-points of $M_{0}$ are given by $M_{0}(F)$
$\quad= \begin{cases}\left\{\operatorname{diag}\left(t_{1}, \ldots, t_{n}, \theta\left(t_{n}\right)^{-1}, \ldots, \theta\left(t_{1}\right)^{-1}\right): t_{i} \in E^{\times}\right\} & \text {for } N=2 n, \\ \left\{\operatorname{diag}\left(t_{1}, \ldots, t_{n}, t, \theta\left(t_{n}\right)^{-1}, \ldots, \theta\left(t_{1}\right)^{-1}\right): t_{i} \in E^{\times}, t \in E^{1}\right\} & \text { for } N=2 n+1 .\end{cases}$ 
Let $A_{0}$ be a maximal $F$-split torus of $U_{N}$, which is a subtorus of $M_{0}$. Then

$$
A_{0}(F)= \begin{cases}\left\{\operatorname{diag}\left(t_{1}, \ldots, t_{n}, t_{n}^{-1}, \ldots, t_{1}^{-1}\right): t_{i} \in F^{\times}\right\} & \text {for } N=2 n, \\ \left\{\operatorname{diag}\left(t_{1}, \ldots, t_{n}, 1, t_{n}^{-1}, \ldots, t_{1}^{-1}\right): t_{i} \in F^{\times}\right\} & \text {for } N=2 n+1 .\end{cases}
$$

The absolute root system $\Phi=\Phi\left(U_{N}, M_{0}\right)$ of $U_{N}$ with respect to $M_{0}$ is of type $A_{N-1}$. The root system $\Phi_{\text {red }}=\Phi\left(U_{N}, A_{0}\right)$ of $U_{N}$ with respect to $A_{0}$ is a reduced root system. It is of type $C_{n}$ for $N=2 n$ and of type $B C_{n}$ for $N=2 n+1$. We make the choice of positive roots according to the fixed Borel subgroup $P_{0}$, and let $\Delta$ be the set of simple roots. We order the simple roots as in Bourbaki [1968].

Let $P$ be the Siegel maximal proper standard parabolic $F$-subgroup of $U_{N}$. That is, it is defined, in a standard fashion, by a subset of simple roots obtained by removing the last simple root in the Bourbaki ordering (cf. [Bourbaki 1968] and [Shahidi 2010, Section 1.2]). Let $P=M_{P} N_{P}$ be the Levi decomposition of $P$, where

$$
M_{P} \cong \begin{cases}G_{n} & \text { for } N=2 n, \\ G_{n} \times U_{1} & \text { for } N=2 n+1,\end{cases}
$$

is the Levi factor, and $N_{P}$ the unipotent radical.

1.B. L-groups. The $L$-group of $U_{N}$ is a semidirect product

$$
{ }^{L} U_{N}=\mathrm{GL}_{N}(\mathbb{C}) \rtimes W_{F},
$$

where $W_{F}$ is the Weil group of $F$. It is acting on the connected component ${ }^{L} U_{N}^{\circ}=$ $\mathrm{GL}_{N}(\mathbb{C})$ through the quotient $W_{F} / W_{E} \cong \mathrm{Gal}(E / F)$. The action of the nontrivial Galois automorphism $\theta \in \mathrm{Gal}(E / F)$ is given by

$$
\theta(g)=J_{N}^{\prime-1}{ }^{t} g^{-1} J_{N}^{\prime}
$$

for all $g \in \mathrm{GL}_{N}(\mathbb{C})$.

The $L$-group of the Levi factor $M_{P}$ is a semidirect product

$$
{ }^{L} M_{P}= \begin{cases}\mathrm{GL}_{n}(\mathbb{C}) \times \mathrm{GL}_{n}(\mathbb{C}) \rtimes W_{F} & \text { for } N=2 n, \\ \mathrm{GL}_{n}(\mathbb{C}) \times \mathrm{GL}_{1}(\mathbb{C}) \times \mathrm{GL}_{n}(\mathbb{C}) \rtimes W_{F} & \text { for } N=2 n+1,\end{cases}
$$

where the Weil group $W_{F}$ acts through the quotient $W_{F} / W_{E} \cong \operatorname{Gal}(E / F)$ on the connected component of the $L$-group, and $\theta \in \operatorname{Gal}(E / F)$ acts by interchanging the two $\mathrm{GL}_{n}(\mathbb{C})$ factors.

\section{Eisenstein series and Asai $L$-functions}

In this section we relate the analytic behavior of the Eisenstein series on the unitary group supported in the Siegel parabolic subgroup to a ratio of the Asai $L$-functions appearing in its constant term. For the study of analytic properties of the Asai $L$ functions, it is sufficient to consider the even quasisplit unitary group $U_{2 n}$. However, 
for completeness and future reference, we also study the Eisenstein series in the odd case.

We retain all the notation of Section 1. So, $P$ is the Siegel maximal proper standard parabolic $F$-subgroup of $U_{N}$, with the Levi factor $M_{P} \cong G_{n}$ if $N=2 n$ is even, and $M_{P} \cong G_{n} \times U_{1}$ if $N=2 n+1$ is odd, and the unipotent radical $N_{P}$. Recall that $G_{n}=\operatorname{Res}_{E / F} \mathrm{GL}_{n}$.

2.A. Asai L-functions. Let $\sigma$ denote a cuspidal automorphic representation of $G_{n}\left(\mathbb{A}_{F}\right) \cong \mathrm{GL}_{n}\left(\mathbb{A}_{E}\right)$ and $v$ a character of $U_{1}\left(\mathbb{A}_{F}\right) \cong \mathbb{A}_{E}^{1}$ trivial on $U_{1}(F) \cong E^{1}$. To make a convenient normalization in the case of odd unitary groups, as in [Rogawski 1990] and [Goldberg 1994, Section 6], we denote by $\hat{v}$ a unitary character of $\mathrm{GL}_{n}\left(\mathbb{A}_{E}\right)$ given by

$$
\hat{v}(g)=v\left(\operatorname{det}\left(g^{*} g^{-1}\right)\right)
$$

for all $g \in \mathrm{GL}_{n}\left(\mathbb{A}_{E}\right)$. Observe that $\operatorname{det}\left(g^{*} g^{-1}\right)$ is of norm one. Then we define a cuspidal automorphic representation $\Sigma$ of the Levi factor $M_{P}\left(\mathbb{A}_{F}\right)$ as

$$
\Sigma= \begin{cases}\sigma & \text { for } N=2 n \\ (\sigma \hat{v}) \otimes v & \text { for } N=2 n+1\end{cases}
$$

More precisely, in the case of odd unitary groups the action of $\Sigma$ is given by

$$
\Sigma(g, t)=\sigma(g) v\left(\operatorname{det}\left(g^{*} g^{-1}\right)\right) v(t)
$$

for $g \in \mathrm{GL}_{n}\left(\mathbb{A}_{E}\right)$ and $t \in \mathbb{A}_{E}^{1}$. We always assume that $\Sigma$ is irreducible unitary and trivial on $A_{P}\left(F_{\infty}\right)^{\circ}$, the identity connected component of $A_{P}\left(F_{\infty}\right)$, where $A_{P}$ is a maximal $F$-split torus in the center of $M_{P}$. The last condition is not restrictive. It is just a convenient normalization, obtained by twisting by a unitary character, which makes the poles of Eisenstein series real.

We define first the local $L$-functions. Let $v$ be a place of $F$. By extension of scalars from $F$ to $F_{v}$, we may view the unitary group $U_{N}$ as an algebraic group over $F_{v}$. This algebraic group is denoted by $U_{N}$. Then we have the parabolic subgroup $P_{v}$ of $U_{N}$ defined over $F_{v}$ with Levi decomposition $M_{P, v} N_{P, v}$, where $M_{P, v}$ is the Levi factor and $N_{P, v}$ the unipotent radical.

In the case of the even unitary group, say $N=2 n$, the adjoint representation $r_{v}$ of the $L$-group ${ }^{L} M_{P, v}$ on the Lie algebra ${ }^{L} \mathfrak{n}_{P, v}$ of the $L$-group of $N_{P, v}$ is irreducible for all places $v$ of $F$. If $v$ does not split in $E$, then $r_{v}$ is called the Asai representation, as it generalizes the case considered by Asai in [1977]. We denote it by $r_{A, v}$. This situation is labeled ${ }^{2} A_{2 n-1}-2$ in the list of [Shahidi 1988, Section 4] and [2010, Appendix C]. Explicit action of $r_{A, v}$ is given in [Goldberg 1994, Section 3].

In the case of the odd unitary group, say $N=2 n+1$, the analogous adjoint representation is a direct sum $r_{1, v} \oplus r_{2, v}$ of two irreducible representations for all 
places $v$ of $F$, ordered as in [Shahidi 1990]. If $v$ does not split in $E$, then $r_{2, v}$ is the twisted Asai representation $r_{A, v} \otimes \delta_{E_{w} / F_{v}}$, where $w$ is the unique place of $E$ lying over $v$. This situation is labeled ${ }^{2} A_{2 n}-3$ in the list of [1988, Section 4] and [2010, Appendix C].

For a cuspidal automorphic representation $\Sigma$ of $M_{P}\left(\mathbb{A}_{F}\right)$, let $\Sigma \cong \otimes_{v}^{\prime} \Sigma_{v}$ be a decomposition into a restricted tensor product over all places. Let $R_{v}$ be one of the adjoint representations defined above. Then the local $L$-functions $L\left(s, \Sigma_{v}, R_{v}\right)$ attached to $\Sigma_{v}$ and $R_{v}$ are defined as follows.

- At archimedean places, they are the Artin $L$-functions attached to the Langlands parameter of $\Sigma_{v}$ as in [Shahidi 1985] (see also [2010, Section 8.2], and [Langlands 1989] where the Langlands parametrization over reals was first introduced).

- At unramified nonarchimedean places, they are given in terms of Satake parameters of $\Sigma_{v}$ (see [Shahidi 1988; 2010, Definition 2.3.5], and also [Harder et al. 1986] where Asai's name came up first).

- At the remaining nonarchimedean places, they are defined using the LanglandsShahidi method [Shahidi 1990, Section 7] (see also [Shahidi 2010, Section 8.4]).

The corresponding global $L$-functions are defined as the analytic continuation from the domain of convergence of the product over all places of local $L$-functions $L\left(s, \Sigma_{v}, R_{v}\right)$. According to [Langlands 1971] (see also [Shahidi 2010, Section 2.5]), the product over all places defining the global $L$-functions converges absolutely in some right half-plane $\operatorname{Re}(s)>C$, where $C$ is sufficiently large.

The global $L$-function obtained in this way from $\Sigma=\sigma \cong \otimes_{v}^{\prime} \sigma_{v}$ and $R_{v}=r_{1, v}$ is denoted by $L\left(s, \sigma, r_{A}\right)$ and called the Asai $L$-function attached to $\sigma$. Its analytic properties are the main concern of this paper.

The global $L$-function obtained from $\Sigma \cong \otimes_{v}^{\prime} \Sigma_{v}$ and $R_{v}=r_{2, v}$ is denoted by $L\left(s, \Sigma, r_{A} \otimes \delta_{E / F}\right)$ and called the twisted Asai $L$-function attached to $\Sigma$. In fact, it is the same as the Asai $L$-function $L\left(s, \sigma \otimes \hat{\delta}, r_{A}\right)$ attached to $\sigma \otimes \hat{\delta}$ (see [Goldberg 1994]). Hence, the analytic properties of the twisted Asai $L$-function follow from the analytic properties of the Asai $L$-function attached to a twisted representation. Recall that $\hat{\delta}$ is any extension of the quadratic character $\delta_{E / F}$ to $\mathbb{A}_{E}^{\times}$.

Finally, as shown in [Goldberg 1994], the choice of the normalization of $\Sigma$ in the case of odd unitary groups implies that the global $L$-function obtained from $\Sigma \cong \otimes_{v}^{\prime} \Sigma_{v}$ and $R_{v}=r_{1, v}$ is the same as the principal $L$-function $L(s, \sigma)$ attached to $\sigma$ by [Godement and Jacquet 1972]. Its analytic properties are well known. It is entire, unless $n=1$ and $\sigma$ is the trivial character $\mathbf{1}_{\mathbb{A}_{E}^{\times}}$of $\mathbb{A}_{E}^{\times}$. In that case $L\left(s, \mathbf{1}_{\mathbb{A}_{E}^{\times}}\right)$ is holomorphic except for simple poles at $s=0$ and $s=1$. 
2.B. Eisenstein series. For $s \in \mathbb{C}$ and $\Sigma$ a cuspidal automorphic representation of $M_{P}\left(\mathbb{A}_{F}\right)$ as above, let

$$
I(s, \Sigma)= \begin{cases}\operatorname{Ind}_{P\left(\mathbb{A}_{F}\right)}^{U_{N}\left(\mathbb{A}_{F}\right)}\left(\sigma|\operatorname{det}|_{E}^{s}\right) & \text { for } N=2 n, \\ \operatorname{Ind}_{P\left(\mathbb{A}_{F}\right)}^{U_{N}\left(\mathbb{A}_{F}\right)}\left(\sigma \hat{v}|\operatorname{det}|_{E}^{s} \otimes v\right) & \text { for } N=2 n+1,\end{cases}
$$

be the induced representation, where the induction is normalized. As in [Shahidi 2010, page 108], we realize $I(s, \Sigma)$ for all $s \in \mathbb{C}$ on the same space $W_{\Sigma}$ of smooth functions

$$
f: N_{P}\left(\mathbb{A}_{F}\right) M_{P}(F) A_{P}\left(F_{\infty}\right)^{\circ} \backslash U_{N}\left(\mathbb{A}_{F}\right) \rightarrow \mathbb{C},
$$

$K$-finite with respect to a fixed maximal compact subgroup $K$ of $U_{N}\left(\mathbb{A}_{F}\right)$ compatible to $P$ (as in [Mœglin and Waldspurger 1995, Section I.1.4]), and such that the function on $M_{P}\left(\mathbb{A}_{F}\right)$ given by the assignment $m \mapsto f(m g)$ for $m \in M_{P}\left(\mathbb{A}_{F}\right)$ belongs to the space of $\Sigma$ for all $g \in U_{N}\left(\mathbb{A}_{F}\right)$. The dependence on $s \in \mathbb{C}$ is hidden in the action of $U_{N}\left(\mathbb{A}_{F}\right)$.

Given $f \in W_{\Sigma}$ and $s \in \mathbb{C}$, set

$$
f_{s}(g)=f(g) \exp \left\langle s+\rho_{P}, H_{P}(g)\right\rangle
$$

for all $g \in U_{N}\left(\mathbb{A}_{F}\right)$. Here $\rho_{P}$ is the half-sum of positive roots not being the roots of $M_{P}$, and $H_{P}$ is a map

$$
H_{P}: U_{N}\left(\mathbb{A}_{F}\right) \rightarrow \operatorname{Hom}\left(X\left(M_{P}\right)_{F}, \mathbb{R}\right),
$$

where $X\left(M_{P}\right)_{F}$ denotes the group of $F$-rational characters of $M_{P}$, defined on $m=\left(m_{v}\right)_{v} \in M_{P}\left(\mathbb{A}_{F}\right)$ by the condition

$$
\exp \left\langle\chi, H_{P}(m)\right\rangle=\prod_{v}\left|\chi\left(m_{v}\right)\right|_{v}
$$

for every $\chi \in X\left(M_{P}\right)_{F}$, and extended via Iwasawa decomposition to $U_{N}\left(\mathbb{A}_{F}\right)$ trivially on the unipotent radical $N_{P}\left(\mathbb{A}_{F}\right)$ and the fixed maximal compact subgroup $K$ (cf. [Shahidi 2010, Section 1.3]). Then the Eisenstein series is defined as the analytic continuation from the domain of convergence $\operatorname{Re}(s)>\rho_{P}$ of the series

$$
E(f, s)(g)=\sum_{\gamma \in P(F) \backslash U_{N}(F)} f(\gamma g) \exp \left\langle s+\rho_{P}, H_{P}(\gamma g)\right\rangle=\sum_{\gamma \in P(F) \backslash U_{N}(F)} f_{s}(\gamma g)
$$

for $g \in U_{N}\left(\mathbb{A}_{F}\right)$. The Eisenstein series $E(f, s)$ has a finite number of simple poles in the real interval $0<s \leq \rho_{P}$, and all other poles have $\operatorname{Re}(s)<0$ (cf. [Mœglin and Waldspurger 1995, Section IV.1.11 and IV.3.12]). The residue of the Eisenstein series $E(f, s)$ at $s>0$ is a square-integrable automorphic form on $U_{N}\left(\mathbb{A}_{F}\right)$, but not cuspidal, thus belonging to the residual spectrum of $U_{N}\left(\mathbb{A}_{F}\right)$. In fact, such residues for all $f \in W_{\Sigma}$ span the summand of the residual spectrum of $U_{N}\left(\mathbb{A}_{F}\right)$ with cuspidal support in $\Sigma$ (see [Mœglin and Waldspurger 1995, Section III.2.6] 
or [Franke and Schwermer 1998, Section 1] for the decomposition of the space of automorphic forms with respect to their cuspidal support).

2.C. Asai L-functions in the constant term. Now we prove that the poles of Eisenstein series $E(f, s)(g)$ for $\operatorname{Re}(s)>0$ coincide with the poles for $\operatorname{Re}(s)>0$ of the ratio of $L$-functions appearing in its constant term.

Theorem 2.1. Let $\sigma$ be a cuspidal automorphic representation of $G_{n}\left(\mathbb{A}_{F}\right) \cong$ $\mathrm{GL}_{n}\left(\mathbb{A}_{E}\right)$ and $v$ a unitary character of $U_{1}\left(\mathbb{A}_{F}\right) \cong \mathbb{A}_{E}^{1}$ trivial on $U_{1}(F) \cong E^{1}$. As in Section 2.B, form a cuspidal automorphic representation $\Sigma$ of the Levi factor $M_{P}\left(\mathbb{A}_{F}\right)$ in $U_{N}$. Then the poles with $\operatorname{Re}(s)>0$ of the Eisenstein series $E(f, s)$ for some $f \in W_{\Sigma}$ coincide with the poles satisfying $\operatorname{Re}(s)>0$ of

$$
\begin{cases}\frac{L\left(2 s, \sigma, r_{A}\right)}{L\left(1+2 s, \sigma, r_{A}\right)} & \text { if } N=2 n, \\ \frac{L(s, \sigma)}{L(1+s, \sigma)} \cdot \frac{L\left(2 s, \sigma \otimes \hat{\delta}, r_{A}\right)}{L\left(1+2 s, \sigma \otimes \hat{\delta}, r_{A}\right)} & \text { if } N=2 n+1,\end{cases}
$$

where $\hat{\delta}$ is any extension to $\mathbb{A}_{E}^{\times} / E^{\times}$of the quadratic character $\delta_{E / F}$ of $\mathbb{A}_{F}^{\times} / F^{\times}$ attached to $E / F$ by class field theory.

Remark 2.2. Observe the factor 2 appearing in the argument $2 s$ of the Asai $L$ function in the case of even unitary groups. The reason is that we have chosen, as in [Shahidi 1992], the determinant character to normalize the identification with $\mathbb{C}$ of the complex parameter $s$ in the Eisenstein series, instead of the character $\tilde{\alpha}$ given in terms of the half-sum of positive roots and the coroot of the unique simple root $\alpha$ not being a root of $M_{P}$, as in [Shahidi 1990].

Proof of Theorem 2.1. This is an application of the Langlands spectral theory, using the Langlands-Shahidi method to normalize the intertwining operator.

The poles of the Eisenstein series $E(f, s)$ coincide with the poles of its constant term $E(f, s)_{P}$ along $P$. The constant term is defined as

$$
E(f, s)_{P}(g)=\int_{N_{P}(F) \backslash N_{P}\left(\mathbb{A}_{F}\right)} E(f, s)(n g) d n,
$$

where $d n$ is a fixed Haar measure on $N_{P}\left(\mathbb{A}_{F}\right)$. On the other hand, the constant term can be written as

$$
E(f, s)_{P}(g)=f_{s}(g)+\left(M\left(s, \Sigma, w_{0}\right) f\right)_{-s}(g),
$$

where $M\left(s, \Sigma, w_{0}\right)$ is the standard intertwining operator. Here $w_{0}$ is the unique nontrivial Weyl group element such that $w_{0}(\alpha)$ is a simple root for all simple roots $\alpha$ except the last one in the ordering of [Bourbaki 1968]. 
As in [Shahidi 2010, page 109], the standard intertwining operator is defined as the analytic continuation from the domain of convergence of the integral

$$
M\left(s, \Sigma, w_{0}\right) f(g)=\left(\int_{N_{P}\left(\mathbb{A}_{F}\right)} f_{s}\left(\dot{w}_{0}^{-1} n g\right) d n\right) \exp \left\langle s-\rho_{P}, H_{P}(g)\right\rangle,
$$

where $\dot{w}_{0}$ is a fixed representative for $w_{0}$ in $U_{N}(F)$. For $s \in \mathbb{C}$ away from poles, the assignment $f \mapsto M\left(s, \Sigma, w_{0}\right) f$ defines a linear map on $W_{\Sigma}$, which depends on $s$. It intertwines the actions of $I(s, \Sigma)$ and $I\left(-s, \Sigma^{w_{0}}\right)$. Let $\sigma^{\theta}$ denote $\sigma$ conjugated by the nontrivial Galois automorphism $\theta \in \operatorname{Gal}(E / F)$, that is, $\sigma^{\theta}(m)=\sigma\left(m^{\theta}\right)$ for all $m \in \mathrm{GL}_{n}\left(\mathbb{A}_{E}\right)$. Note that in our case the conjugation by $w_{0}$ amounts to taking $\tilde{\sigma}^{\theta}$, where $\tilde{\sigma}$ is the contragredient of $\sigma$. In the case of odd unitary groups this means that $\Sigma^{w_{0}} \cong \tilde{\sigma}^{\theta} \hat{v} \otimes v$ (see [Goldberg 1994]).

It is clear from the expression for the constant term that the poles of the Eisenstein series are the same as those of the standard intertwining operator. We apply the Langlands-Shahidi method to normalize this operator. The normalizing factor in this situation, labeled ${ }^{2} A_{2 n-1}-2$ for the even unitary group and ${ }^{2} A_{2 n}-3$ for the odd unitary group in the list of [Shahidi 1988, Section 4] and [2010, Appendix C], is given in terms of $L$-functions and corresponding $\varepsilon$-factors as

$$
\begin{aligned}
r(s, & \left.\Sigma, w_{0}\right) \\
& = \begin{cases}\frac{L\left(2 s, \sigma, r_{A}\right)}{L\left(1+2 s, \sigma, r_{A}\right) \varepsilon\left(2 s, \sigma, r_{A}\right)} & \text { for } N=2 n, \\
\frac{L(s, \sigma)}{L(1+s, \sigma) \varepsilon(s, \sigma)} \cdot \frac{L\left(2 s, \sigma \otimes \hat{\delta}, r_{A}\right)}{L\left(1+2 s, \sigma \otimes \hat{\delta}, r_{A}\right) \varepsilon\left(2 s, \sigma \otimes \hat{\delta}, r_{A}\right)} & \text { for } N=2 n+1 .\end{cases}
\end{aligned}
$$

The normalized intertwining operator

$$
r\left(s, \Sigma, w_{0}\right)^{-1} M\left(s, \Sigma, w_{0}\right)
$$

is holomorphic and not identically vanishing on $I(s, \Sigma)$ for $\operatorname{Re}(s)>0$. This is essentially a local fact proved in Lemma 2.3 below.

Assuming this fact, we now finish the proof. The holomorphy and nonvanishing of the normalized operator implies that the poles of $M\left(s, \Sigma, w_{0}\right)$ for $\operatorname{Re}(s)>0$ coincide with those of $r\left(s, \Sigma, w_{0}\right)$. Since the $\varepsilon$-factors are entire and nonvanishing for all $s \in \mathbb{C}$, these are the same as the poles of the ratios of $L$-functions given in the theorem.

2.D. Holomorphy and nonvanishing of normalized intertwining operators. It remains to show the fact that $r\left(s, \Sigma, w_{0}\right)^{-1} M\left(s, \Sigma, w_{0}\right)$ is holomorphic and nonvanishing for $\operatorname{Re}(s)>0$. The notation is as in the proof of the previous theorem. This is essentially a local problem, because one can decompose over the places of $F$ the action of the standard intertwining operator acting on a decomposable function using the fact that all ingredients are unramified at all but finitely many places. 
Hence, the problem reduces to a finite number of ramified and archimedean places, which is solved for each place separately.

We introduce some local notation first. Let $\Sigma \cong \otimes_{v}^{\prime} \Sigma_{v}$ be the decomposition of $\Sigma$ into a restricted tensor product, where in the case of odd unitary groups $\Sigma_{v}=\sigma_{v} \hat{\nu}_{v} \otimes v_{v}$. We denote the local standard intertwining operator by $M\left(s, \Sigma_{v}, w_{0}\right)$. It is defined as the analytic continuation of the local analogue of the integral defining the global operator $M\left(s, \Sigma, w_{0}\right)$ (see the proof of Theorem 2.1). Let $r\left(s, \Sigma_{v}, w_{0}\right)$ be the local factor at $v$ of $r\left(s, \Sigma, w_{0}\right)$. We show in the lemma below that the normalized local intertwining operator

$$
N\left(s, \Sigma_{v}, w_{0}\right)=r\left(s, \Sigma_{v}, w_{0}\right)^{-1} M\left(s, \Sigma_{v}, w_{0}\right)
$$

is holomorphic and not identically vanishing on the local induced representation $I\left(s, \Sigma_{v}\right)$ for $\operatorname{Re}(s)>0$.

Lemma 2.3. Let $\Sigma_{v}$ be a local component of a cuspidal automorphic representation $\Sigma$ of the Levi factor $M_{P}\left(\mathbb{A}_{F}\right)$ in the unitary group $U_{N}$. Then, for $\operatorname{Re}(s)>0$, the normalized local intertwining operator $N\left(s, \Sigma_{v}, w_{0}\right)$ is holomorphic and not identically vanishing on the induced representation $I\left(s, \Sigma_{v}\right)$.

Proof. Consider first the case in which the place $v$ of $F$ splits in $E$. Then $U_{N}\left(F_{v}\right)$ is isomorphic to $\mathrm{GL}_{N}\left(F_{v}\right)$, and the Levi factor

$$
M_{P}\left(F_{v}\right) \cong \begin{cases}\mathrm{GL}_{n}\left(F_{v}\right) \times \mathrm{GL}_{n}\left(F_{v}\right) & \text { for } N=2 n \\ \mathrm{GL}_{n}\left(F_{v}\right) \times \mathrm{GL}_{1}\left(F_{v}\right) \times \mathrm{GL}_{n}\left(F_{v}\right) & \text { for } N=2 n+1 .\end{cases}
$$

Hence, the normalized operator considered in the lemma is attached to a unitary representation of a Levi factor $M_{P}\left(F_{v}\right)$ in $\mathrm{GL}_{N}\left(F_{v}\right)$. The holomorphy and nonvanishing for $\operatorname{Re}(s)>0$ follow from [Mœglin and Waldspurger 1989, Proposition I.10].

We consider now the case in which the place $v$ of $F$ does not split in $E$, and denote by $w$ the unique place of $E$ lying over $v$. Then $E_{w} / F_{v}$ is a quadratic extension of local fields, and $U_{N}\left(F_{v}\right)$ is the quasisplit unitary group in $N$ variables given by the extension $E_{w} / F_{v}$. The Levi factor $M_{P}\left(F_{v}\right)$ is isomorphic to

$$
M_{P}\left(F_{v}\right) \cong \begin{cases}G_{n}\left(F_{v}\right) \cong \mathrm{GL}_{n}\left(E_{w}\right) & \text { for } N=2 n, \\ G_{n}\left(F_{v}\right) \times U_{1}\left(F_{v}\right) \cong \mathrm{GL}_{n}\left(E_{w}\right) \times E_{w}^{1} & \text { for } N=2 n+1,\end{cases}
$$

so that

$$
\Sigma_{v} \cong \begin{cases}\sigma_{w} & \text { for } N=2 n \\ \left(\sigma_{w} \hat{v}_{w}\right) \otimes v_{w} & \text { for } N=2 n+1,\end{cases}
$$

where $\sigma_{w}$ is the local component of a cuspidal automorphic representation $\sigma$ of $\mathrm{GL}_{n}\left(\mathbb{A}_{E}\right)$ at the place $w$ of $E$, and $v_{w}$ the local component of a unitary character $v$ of $\mathbb{A}_{E}^{1}$ trivial on $E^{1}$. 
In particular, $\sigma_{w}$ is unitary and generic, since it is a local component of a cuspidal automorphic representation of $\mathrm{GL}_{n}\left(\mathbb{A}_{E}\right)$. Hence, by [Tadić 1986] and [Vogan 1986], in the nonarchimedean and archimedean cases, respectively, there is

- a standard parabolic subgroup $Q$ of $\mathrm{GL}_{n}$ such that the Levi factor $M_{Q}$ of $Q$ is isomorphic to $\mathrm{GL}_{d_{1}} \times \cdots \times \mathrm{GL}_{d_{\ell}}$, where $d_{1}+\cdots+d_{\ell}=n$,

- unitary square-integrable representations $\delta_{i}$ of $\mathrm{GL}_{d_{i}}\left(E_{w}\right)$, for $i=1, \ldots, \ell$, and

- real numbers $\alpha_{i}$ with $0 \leq\left|\alpha_{i}\right|<\frac{1}{2}$, for $i=1, \ldots, \ell$,

such that $\sigma_{w}$ is isomorphic to the fully induced representation

$$
\sigma_{w} \cong \operatorname{Ind}_{Q\left(E_{w}\right)}^{\mathrm{GL}_{n}\left(E_{w}\right)}\left(\delta_{1}|\operatorname{det}|^{\alpha_{1}} \otimes \cdots \otimes \delta_{\ell}|\operatorname{det}|^{\alpha_{\ell}}\right) .
$$

Let $R$ be the standard parabolic $F$-subgroup of $U_{N}$ with the Levi factor

$$
M_{R} \cong \begin{cases}G_{d_{1}} \times \cdots \times G_{d_{\ell}} & \text { for } N=2 n \\ G_{d_{1}} \times \cdots \times G_{d_{\ell}} \times U_{1} & \text { for } N=2 n+1,\end{cases}
$$

so that $R \subset P$ and $M_{R}\left(F_{v}\right)=M_{Q}\left(E_{w}\right)$ for $N=2 n$ and $M_{R}\left(F_{v}\right)=M_{Q}\left(E_{w}\right) \times E_{w}^{1}$ for $N=2 n+1$. Let

$$
\delta= \begin{cases}\delta_{1} \otimes \cdots \otimes \delta_{\ell} & \text { for } N=2 n, \\ \delta_{1} \hat{v}_{1} \otimes \cdots \otimes \delta_{\ell} \hat{v}_{\ell} \otimes v & \text { for } N=2 n+1,\end{cases}
$$

be a unitary square-integrable representation of $M_{R}\left(F_{v}\right)$, where $\hat{v}_{i}$ is the character of $\mathrm{GL}_{d_{i}}\left(E_{w}\right)$ given by $\hat{v}_{i}\left(h_{i}\right)=v\left(\operatorname{det}\left(h_{i}{ }^{*} h_{i}^{-1}\right)\right)$ for $h_{i} \in \mathrm{GL}_{d_{i}}\left(E_{w}\right)$.

By induction in stages, the intertwining operator $N\left(s, \Sigma_{v}, w_{0}\right)$ coincides with the intertwining operator

$$
N\left(\left(s+\alpha_{1}, \ldots, s+\alpha_{\ell}\right), \delta, w_{0}\right)
$$

acting on the induced representation

$$
\begin{cases}\operatorname{Ind}_{R\left(F_{v}\right)}^{U_{N}\left(F_{v}\right)}\left(\delta_{1}|\operatorname{det}|^{s+\alpha_{1}} \otimes \cdots \otimes \delta_{\ell}|\operatorname{det}|^{s+\alpha_{\ell}}\right) & \text { for } N=2 n, \\ \operatorname{Ind}_{R\left(F_{v}\right)}^{U_{N}\left(F_{v}\right)}\left(\delta_{1} \hat{v}_{1}|\operatorname{det}|^{s+\alpha_{1}} \otimes \cdots \otimes \delta_{\ell} \hat{v}_{\ell}|\operatorname{det}|^{s+\alpha_{\ell}} \otimes v\right) & \text { for } N=2 n+1 .\end{cases}
$$

By Zhang's lemma [1997] (see also [Kim 2000, Lemma 1.7]), the holomorphy of this last operator at $s$ implies nonvanishing. Hence, to show the lemma, it is sufficient to prove the holomorphy for $\operatorname{Re}(s)>0$.

To prove the holomorphy for $\operatorname{Re}(s)>0$, we decompose the intertwining operator into a product of intertwining operators as in [Shahidi 1981, Section 2.1]. If we show that each factor is holomorphic for $\operatorname{Re}(s)>0$, then the product is holomorphic for $\operatorname{Re}(s)>0$ as well, and the lemma is proved. The factors are normalized intertwining operators that can be viewed as intertwining operators on representations induced from appropriate maximal proper parabolic subgroups in certain reductive groups. 
In our case these rank-one factors are normalized operators

$$
N\left(2 s+\alpha_{i}+\alpha_{j}, \delta_{i} \otimes \tilde{\delta}_{j}^{\theta}\right),
$$

for $1 \leq i<j \leq \ell$, acting on the induced representations

$$
\operatorname{Ind}_{Q_{i, j}\left(E_{w}\right)}^{\mathrm{GL}_{d_{i}+d_{j}}\left(E_{w}\right)}\left(\delta_{i}|\operatorname{det}|^{s+\alpha_{i}} \otimes \tilde{\delta}_{j}^{\theta}|\operatorname{det}|^{-s-\alpha_{j}}\right),
$$

where $Q_{i, j}$ is the maximal standard proper parabolic subgroup of $\mathrm{GL}_{d_{i}+d_{j}}$ with the Levi factor $\mathrm{GL}_{d_{i}} \times \mathrm{GL}_{d_{j}}$, and normalized operators

$$
\begin{cases}N\left(s+\alpha_{k}, \delta_{k}\right) & \text { for } N=2 n, \\ N\left(s+\alpha_{k},\left(\delta_{k} \hat{v}_{k}\right) \otimes v\right) & \text { for } N=2 n+1,\end{cases}
$$

for $1 \leq k \leq \ell$, acting on the induced representation

$$
\begin{cases}\operatorname{Ind}_{Q_{k}\left(F_{v}\right)}^{U_{2 d_{k}}\left(F_{v}\right)}\left(\delta_{k}|\operatorname{det}|^{s+\alpha_{k}}\right) & \text { for } N=2 n, \\ \operatorname{Ind}_{Q_{k}\left(F_{v}\right)}^{U_{2 d_{k}+1}\left(F_{v}\right)}\left(\delta_{k} \hat{v}_{k}|\operatorname{det}|^{s+\alpha_{k}} \otimes v\right) & \text { for } N=2 n+1,\end{cases}
$$

where $Q_{k}$ is the maximal standard proper parabolic subgroup of $U_{2 d_{k}}$ with the Levi factor $G_{d_{k}}$ if $N=2 n$, and of $U_{2 d_{k}+1}$ with the Levi factor $G_{d_{k}} \times U_{1}$ if $N=2 n+1$. We suppress the Weyl group element from the notation for these intertwining operators, because they are always determined by the maximal parabolic subgroup in question.

According to [Zhang 1997, Section 2], the rank-one normalized intertwining operator is holomorphic when the real part of its complex parameter is greater than the first negative point of reducibility of the induced representation on which it acts. For $\operatorname{Re}(s)>0$, using the bound on $\alpha_{i}$, we have

$$
\operatorname{Re}\left(s+\alpha_{i}+\alpha_{j}\right)>-1 \text { and } \operatorname{Re}\left(s+\alpha_{k}\right)>-\frac{1}{2} .
$$

But these two bounds are precisely the first negative points of reducibility in the cases $Q_{i, j} \subset \mathrm{GL}_{d_{i}+d_{j}}$ and $Q_{k} \subset U_{2 d_{k}}$ or $U_{2 d_{k}+1}$. This essentially follows from the standard module conjecture, proved in [Vogan 1978] for any quasisplit real group, and thus for complex groups as well, and in [Muić 2001] for quasisplit classical groups over a $p$-adic field. In [Casselman and Shahidi 1998, Section 5] the reducibility points are determined in terms of local coefficients over any local field. A convenient reference making explicit the first reducibility points of such complementary series using local coefficients for any quasisplit classical group over a local field of characteristic zero is [Lapid et al. 2004, Lemma 2.6 and 2.7]. For the general linear group the reducibility is obtained in [Zelevinsky 1980] over a p-adic field, in [Speh 1981] over reals, and in [Wallach 1979] over complex numbers (see also [Kim 2000, Lemma 2.10]). For the unitary group over a nonarchimedean field, it is obtained in [Goldberg 1994, Section 3 and 6] by applying the general reducibility result of [Shahidi 1990], while at an archimedean place, the $L$-functions 
in the local coefficient that control reducibility are the $L$-functions of the restriction to $\mathbb{R}^{\times}$of a character of $\mathbb{C}^{\times}$(see [Lapid et al. 2004, Lemma 2.6]). Thus, the rank-one factors are all holomorphic and the lemma is proved.

Remark 2.4. Kim and Krishnamurthy [2004; 2005] have proved the holomorphy and nonvanishing of normalized intertwining operators for a representation of the Levi factor of any maximal proper parabolic subgroup of $U_{N}$, which is a local component of a generic cuspidal automorphic representation. Since in our case all cuspidal automorphic representations of the Levi factor are generic, Lemma 2.3 follows from their work. Their proof uses their stable base change lift and bounds towards the Ramanujan conjecture obtained by Luo, Rudnick and Sarnak [1999] to bound the exponents on the unitary group. In our case these bounds are not required because our unitary factor in the Levi is either trivial or rank zero. This simplifies the proof.

\section{Arthur parameters for unitary groups}

Our next task is to introduce the notion of Arthur parameters and the endoscopic classification of automorphic representations for the quasisplit unitary group $U_{N}$ in $N$ variables. We consider both the even and odd case for completeness, although for the application to the analytic properties of the Asai $L$-functions only the even case is required.

In [Mok 2015], the results of [Arthur 2013] (see also [Arthur 2005]) are extended to the case of quasisplit unitary groups. As in [Arthur 2004], we avoid the conjectural Langlands group by describing the parameters in terms of irreducible constituents of the discrete spectrum of general linear groups. For quasisplit classical groups this approach was taken in [Mœglin 2008].

3.A. Arthur parameters. Let $\mu$ be a Galois self-dual cuspidal automorphic representation of $\mathrm{GL}_{m}\left(\mathbb{A}_{E}\right)$. One of the crucial results in Mok's proof of endoscopic classification of representations in the discrete spectrum for quasisplit unitary groups is the uniqueness (up to equivalence) of the twisted endoscopic datum associated to $\mu$. This is the content of [Mok 2015, Theorem 2.4.2]. In fact, this unique endoscopic datum is simple, thus, determining a unique sign $\kappa \in\{ \pm 1\}$ attached to $\mu$. The parity of the endoscopic datum associated to $\mu$ is then defined as $\kappa(-1)^{m-1}$ (as in Section 2.4 of the same reference). Using parity we make the following definition as in [ibid., Theorem 2.5.4] (see also [Gan et al. 2012]).

Definition 3.1. Let $\mu$ be a Galois self-dual cuspidal automorphic representation of $\mathrm{GL}_{m}\left(\mathbb{A}_{E}\right)$. We say that $\mu$ is Galois orthogonal (resp. Galois symplectic), if the parity of the unique twisted endoscopic datum associated to $\mu$ is +1 (resp. -1 ). 
It turns out, as also proved by Mok, that this definition can be rephrased in terms of poles at $s=1$ of the Asai $L$-function $L\left(s, \mu, r_{A}\right)$ attached to $\mu$.

Theorem 3.2 [Mok 2015, Theorem 2.5.4(a)]. Let $\mu$ be a Galois self-dual cuspidal automorphic representation of $\mathrm{GL}_{m}\left(\mathbb{A}_{E}\right)$. Then $\mu$ is Galois orthogonal (resp. Galois symplectic) if and only if the Asai L-function $L\left(s, \mu, r_{A}\right)$ (resp. the twisted Asai $L$-function $\left.L\left(s, \mu \otimes \hat{\delta}, r_{A}\right)\right)$ has a pole at $s=1$, where $\hat{\delta}$ is any extension to $\mathbb{A}_{E}^{\times} / E^{\times}$ of the quadratic character $\delta_{E / F}$ of $\mathbb{A}_{F}^{\times} / F^{\times}$attached to $E / F$ by class field theory.

We are now ready to define global Arthur parameters for the quasisplit unitary group $U_{N}$ in $N$ variables. We in fact define the square-integrable Arthur parameters, which, according to [ibid., Theorem 2.5.2], parameterize global Arthur packets contributing to the discrete automorphic spectrum of $U_{N}\left(\mathbb{A}_{F}\right)$. These parameters depend on the choice of certain character of $\mathbb{A}_{E}^{\times}$, trivial on $E^{\times}$, that defines an $L$-embedding of the $L$-group of $U_{N}$ into the $L$-group of $G_{N}$ (cf. [ibid., Section 2.1]). Roughly speaking, this character determines whether we view parameters as the stable or twisted base change of a representation in the discrete spectrum. Of course, the decomposition of the discrete spectrum is independent of that choice, and we take it in this paper to be the trivial character of $\mathbb{A}_{E}^{\times}$, and suppress it from notation (see [ibid., Theorem 2.5.2]). The reason why Mok considers all possible characters is that they are all required for the induction argument in the proof of endoscopic classification.

Definition 3.3 (Arthur parameters). As before, let $U_{N}$ be the quasisplit unitary group in $N$ variables given by a quadratic extension $E / F$ of number fields. The set $\Psi_{2}\left(U_{N}\right)$ of square-integrable global Arthur parameters for $U_{N}$ is defined as the set of all unordered formal sums of formal tensor products of the form

$$
\psi=\left(\mu_{1} \otimes v\left(n_{1}\right)\right) \boxplus \cdots \boxplus\left(\mu_{\ell} \otimes v\left(n_{\ell}\right)\right),
$$

such that

(i) $\mu_{i}$ is a Galois self-dual cuspidal automorphic representation of $\operatorname{GL}_{m_{i}}\left(\mathbb{A}_{E}\right)$, that is, $\mu_{i} \cong \tilde{\mu}_{i}^{\theta}$;

(ii) $n_{i}$ is a positive integer, and $v\left(n_{i}\right)$ is the unique $n_{i}$-dimensional irreducible algebraic representation of $S L_{2}(\mathbb{C})$;

(iii) $m_{1} n_{1}+\cdots+m_{\ell} n_{\ell}=N$;

(iv) for $i \neq j$, we have $\mu_{i} \nsucceq \mu_{j}$ or $n_{i} \neq n_{j}$, that is, the formal sum $\psi$ is multiplicity free;

(v) representation $\mu_{i}$ is Galois orthogonal (resp. Galois symplectic) if and only if integers $n_{i}$ and $N$ are of the same parity (resp. different parity).

According to Theorem 3.2, condition (v) is equivalent to the condition 
( $\left.\mathrm{v}^{\prime}\right)$ representation $\mu_{i}$ is such that the Asai $L$-function $L\left(s, \mu_{i}, r_{A}\right)$ (resp. the twisted Asai $L$-function $\left.L\left(s, \mu_{i} \otimes \hat{\delta}, r_{A}\right)\right)$ has a pole at $s=1$ if and only if integers $n_{i}$ and $N$ are of the same parity (resp. different parity).

3.B. Arthur packets. We proceed, following [Mok 2015], to define the local and global Arthur packet associated to a global Arthur parameter $\psi \in \Psi_{2}\left(U_{N}\right)$. Every global Arthur parameter $\psi \in \Psi_{2}\left(U_{N}\right)$ gives rise, as in [ibid., Section 2.3], to a local Arthur parameter $\psi_{v}$ for every place $v$ of $F$. The local Arthur packet $\Pi_{\psi_{v}}$ is a finite multiset of unitary irreducible representations of $U_{N}\left(F_{v}\right)$ associated to $\psi_{v}$ in [ibid., Theorem 2.5.1] and the discussion following it. There is a canonical mapping from $\Pi_{\psi_{v}}$ to the character group of a certain finite group $\mathscr{S}_{\psi_{v}}$ attached to $\psi_{v}$ (for a definition see [ibid., Section 2.2]). For $\pi_{v} \in \Pi_{\psi_{v}}$, we denote the corresponding character by $\eta_{\pi_{v}}$. If $U_{N}\left(F_{v}\right)$ and $\pi_{v}$ are unramified, then $\eta_{\pi_{v}}$ is the trivial character. We are skipping here the details, because our main interest is only in unramified places.

The global Arthur packet $\Pi_{\psi}$ associated to $\psi \in \Psi_{2}\left(U_{N}\right)$ is defined as

$$
\Pi_{\psi}=\left\{\otimes_{v}^{\prime} \pi_{v}: \pi_{v} \in \Pi_{\psi_{v}} \text { and } \eta_{\pi_{v}} \text { is trivial for almost all } v\right\} .
$$

The global packets $\Pi_{\psi}$ for all $\psi \in \Psi_{2}\left(U_{N}\right)$ contain all representations that can possibly appear in the decomposition of the discrete spectrum on $U_{N}\left(\mathbb{A}_{F}\right)$. There is a subtle further condition identifying elements of $\Pi_{\psi}$ that indeed appear in the discrete spectrum (for a precise formulation see [Mok 2015, Theorem 2.5.2]). We do not recall this condition, because for our purposes it is sufficient to work with the full packets $\Pi_{\psi}$.

We now compare a representation in the discrete spectrum on $U_{N}\left(\mathbb{A}_{F}\right)$ and its Arthur parameter at unramified places. Through the application to residual representations supported in the Siegel maximal parabolic subgroup, this turns out to be crucial for the proof of holomorphy of the Asai $L$-function inside the critical strip. Given

$$
\psi=\left(\mu_{1} \otimes v\left(n_{1}\right)\right) \boxplus \cdots \boxplus\left(\mu_{\ell} \otimes v\left(n_{\ell}\right)\right) \in \Psi_{2}\left(U_{N}\right),
$$

with notation as in Definition 3.3, let $S$ be a finite set of places of $F$, containing all archimedean places and all nonarchimedean places ramified in $E$, and such that for all places $w$ of $E$ lying above some $v \notin S$ all $\mu_{i, w}$ are unramified. Then, for $v \notin S$, we attach to $\psi$ a Frobenius-Hecke conjugacy class

$$
c_{v}(\psi)= \begin{cases}\bigoplus_{i=1}^{\ell}\left(c\left(\mu_{i, w}\right) \otimes c_{w}\left(v\left(n_{i}\right)\right)\right) & \text { if } v \text { is inert and } v \mid w, \\ \left(\bigoplus_{i=1}^{\ell}\left(c\left(\mu_{i, w_{1}}\right) \otimes c_{w_{1}}\left(v\left(n_{i}\right)\right)\right), \bigoplus_{i=1}^{\ell}\left(c\left(\mu_{i, w_{2}}\right) \otimes c_{w_{2}}\left(v\left(n_{i}\right)\right)\right)\right) & \text { if } v \text { splits into } w_{1}, w_{2},\end{cases}
$$


viewed as a semisimple conjugacy class in the $L$-group of $G_{N}$ over $F_{v}$, where $c\left(\mu_{i, w}\right) \in \mathrm{GL}_{m_{i}}(\mathbb{C})$ is the Satake parameter, and

$$
c_{w}\left(v\left(n_{i}\right)\right)=\operatorname{diag}\left(q_{w}^{\left(n_{i}-1\right) / 2}, q_{w}^{\left(n_{i}-3\right) / 2}, \ldots, q_{w}^{-\left(n_{i}-1\right) / 2}\right),
$$

with $q_{w}$ the cardinality of the residue field of $E_{w}$. Observe that $q_{w}=q_{v}^{2}$ if $v$ is inert in $E$, and $q_{w_{1}}=q_{w_{2}}=q_{v}$ if $v$ splits in $E$. The conjugacy classes $c_{v}(\psi)$ for $v \notin S$ may be viewed as the Satake parameters of the unramified constituents at places $w$ of $E$ lying above $v$ of the induced representation

$$
\begin{array}{r}
\operatorname{Ind}_{R\left(A_{E}\right)}^{\mathrm{GL}_{N}\left(\mathbb{A}_{E}\right)}\left(\mu_{1}|\operatorname{det}|^{\left(n_{1}-1\right) / 2} \otimes \mu_{1}|\operatorname{det}|^{\left(n_{1}-3\right) / 2} \otimes \cdots \otimes \mu_{1}|\operatorname{det}|^{-\left(n_{1}-1\right) / 2}\right. \\
\otimes \mu_{2}|\operatorname{det}|^{\left(n_{2}-1\right) / 2} \otimes \mu_{2}|\operatorname{det}|^{\left(n_{2}-3\right) / 2} \otimes \cdots \otimes \mu_{2}|\operatorname{det}|^{-\left(n_{2}-1\right) / 2} \otimes \cdots \\
\left.\otimes \mu_{\ell}|\operatorname{det}|^{\left(n_{\ell}-1\right) / 2} \otimes \mu_{\ell}|\operatorname{det}|^{\left(n_{\ell}-3\right) / 2} \otimes \cdots \otimes \mu_{\ell}|\operatorname{det}|^{-\left(n_{\ell}-1\right) / 2}\right),
\end{array}
$$

where $R$ is the standard parabolic subgroup of $\mathrm{GL}_{N}$ with the Levi factor $\mathrm{GL}_{m_{1}} \times$ $\cdots \times \mathrm{GL}_{m_{1}} \times \mathrm{GL}_{m_{2}} \times \cdots \times \mathrm{GL}_{m_{2}} \times \cdots \times \mathrm{GL}_{m_{\ell}} \times \cdots \times \mathrm{GL}_{m_{\ell}}$ with $n_{i}$ copies of $\mathrm{GL}_{m_{i}}$ in the product, and $\mu_{i}$ are unramified at $v$.

On the other hand, let $\pi \cong \otimes_{v}^{\prime} \pi_{v}$ be an irreducible automorphic representation appearing in the discrete spectrum on $U_{N}\left(\mathbb{A}_{F}\right)$. Let $S^{\prime}$ be a finite set of places of $F$, containing all archimedean places, and such that for $v \notin S^{\prime}$, we have that $U_{N}\left(F_{v}\right)$ and $\pi_{v}$ are unramified. Then, for $v \notin S^{\prime}$, the Satake isomorphism gives a Frobenius-Hecke conjugacy class $c\left(\pi_{v}\right)$ in the local $L$-group of $U_{N}$ over $F_{v}$. However, we may view $c\left(\pi_{v}\right)$ as a conjugacy class in the local $L$-group of $G_{N}$ through the stable base change map of $L$-groups. This is consistent with our choice of the trivial character in the definition of Arthur parameters.

According to the preliminary comparison of spectral sides of the trace formulas for $U_{N}$ and the twisted trace formula for $\mathrm{GL}_{N}$, carried out in [Mok 2015, Section 4.3] (see also [Arthur 2013, Section 3.4]), for every irreducible automorphic representation $\pi$ of $U_{N}\left(\mathbb{A}_{F}\right)$ appearing in the discrete spectrum, there is a unique corresponding parameter $\psi \in \Psi_{2}\left(U_{N}\right)$ such that the Frobenius-Hecke conjugacy classes $c_{v}(\psi)$ attached to $\psi$ coincide at almost all places with the classes $c\left(\pi_{v}\right)$ attached to $\pi$. This observation is the key to the following proposition.

Remark 3.4. Strictly speaking the preliminary comparison of trace formulas gives unique $\psi$ in a larger set of parameters $\Psi\left(U_{N}\right)$ (see [Mok 2015] for a definition), but the full proof of endoscopic classification shows that such $\psi$ belongs to $\Psi_{2}\left(U_{N}\right)$.

Proposition 3.5. Let $P$ be the Siegel maximal proper parabolic $F$-subgroup of $U_{2 n}$. Let $\sigma$ be a cuspidal automorphic representation of its Levi factor

$$
M_{P}\left(\mathbb{A}_{F}\right) \cong \mathrm{GL}_{n}\left(\mathbb{A}_{\mathbb{E}}\right)
$$


If the induced representation

$$
\operatorname{Ind}_{P\left(\mathbb{A}_{F}\right)}^{U_{2 n}\left(\mathrm{~A}_{F}\right)}\left(\sigma \otimes|\operatorname{det}|_{\AA_{E}}^{S}\right)
$$

has a constituent in the discrete spectrum of $U_{2 n}\left(\mathbb{A}_{F}\right)$ for some $s>0$, then its Arthur parameter is

$$
\psi=\sigma \otimes v(2),
$$

and in particular $s=\frac{1}{2}$ and $\sigma$ is Galois self-dual.

Proof. Since an automorphic representation is unramified at almost all places, the local component of an irreducible constituent $\pi$ of the induced representation

$$
\operatorname{Ind}_{P\left(\AA_{F}\right)}^{U_{2 n}\left(\AA_{F}\right)}\left(\sigma \otimes|\operatorname{det}|_{\AA_{E}}^{S}\right)
$$

belonging to the discrete spectrum is the unramified representation with the Satake parameter, viewed as a conjugacy class in the $L$-group of $G_{2 n}$ as above,

$$
\begin{aligned}
& c\left(\pi_{v}\right) \\
& \quad= \begin{cases}c\left(\sigma_{w}\right) \otimes \operatorname{diag}\left(q_{w}^{s}, q_{w}^{-s}\right) & \text { if } v \text { is inert and } v \mid w, \\
\left(c\left(\sigma_{w_{1}}\right) \otimes \operatorname{diag}\left(q_{w_{1}}^{s}, q_{w_{1}}^{-s}\right), c\left(\sigma_{w_{2}}\right) \otimes \operatorname{diag}\left(q_{w_{2}}^{s}, q_{w_{2}}^{-s}\right)\right) & \text { if } v \text { splits into } w_{1}, w_{2},\end{cases}
\end{aligned}
$$

for almost all places $v$ of $F$. Recall that $q_{w}=q_{v}^{2}$ if $v$ is inert, and $q_{w_{1}}=q_{w_{2}}=q_{v}$ if $v$ splits. We may also view $c\left(\pi_{v}\right)$ as the Satake parameter of the unramified constituent of the local components at places $w$ of $E$ lying over $v$ of the induced representation

$$
\operatorname{Ind}_{Q\left(\AA_{E}\right)}^{\mathrm{GL}_{2 n}\left(\mathbb{A}_{E}\right)}\left(\sigma|\operatorname{det}|_{\AA_{E}}^{s} \otimes \sigma|\operatorname{det}|_{\mathbb{A}_{E}}^{-s}\right),
$$

where $Q$ is the standard parabolic subgroup of $\mathrm{GL}_{2 n}$ with the Levi factor $\mathrm{GL}_{n} \times \mathrm{GL}_{n}$.

By the observation made just before the statement of the proposition, these Frobenius-Hecke conjugacy classes $c\left(\pi_{v}\right)$, viewed as conjugacy classes in the $L$ group of $G_{2 n}$, should match at almost all places the conjugacy classes $c_{v}(\psi)$ attached to the Arthur parameter $\psi \in \Psi_{2}\left(U_{N}\right)$ parameterizing $\pi$. As mentioned above, these $c_{v}(\psi)$ may be viewed as Satake parameters of the unramified constituent at $v$ of certain induced representation of $\mathrm{GL}_{2 n}\left(\mathbb{A}_{E}\right)$. However, by the strong multiplicity one for general linear groups [Jacquet and Shalika 1981, Theorem 4.4], matching of Satake parameters at almost all places for induced representations of $\mathrm{GL}_{N}\left(\mathbb{A}_{E}\right)$ implies that the inducing data for these representations are associate. Since $Q$ is self associate, this means that the parabolic subgroup $R$ determined by $\psi$ as above must be $Q$, and thus that $\psi$ is of the form

$$
\psi=\sigma \otimes v(k),
$$

where $k=2 s+1$. Since $k=2$ by condition (iii) in Definition 3.3, it follows that $s=\frac{1}{2}$. As $\sigma$ appears in $\psi$ it is necessarily Galois self-dual. 


\section{Holomorphy and nonvanishing of Asai $L$-functions}

In this section we prove the analytic properties of the Asai $L$-functions as a consequence of Mok's endoscopic classification [2015] of automorphic representations of a quasisplit unitary group.

4.A. Analytic properties of Eisenstein series. The first task is to determine the poles of Eisenstein series $E(f, s)$ for $\operatorname{Re}(s)>0$. We now consider only the case of even quasisplit unitary group $U_{2 n}$.

Recall that for a cuspidal automorphic representation $\sigma$ of $\mathrm{GL}_{n}\left(\mathbb{A}_{E}\right)$, we let $\sigma^{\theta}$ denote $\sigma$ conjugated by the nontrivial Galois automorphism $\theta \in \operatorname{Gal}(E / F)$. We say that $\sigma$ is Galois self-dual if it is isomorphic to $\tilde{\sigma}^{\theta}$, where $\tilde{\sigma}$ is the contragredient of $\sigma$.

Theorem 4.1. Let $\sigma$ be a cuspidal automorphic representation of the Levi factor $M_{P}\left(\mathbb{A}_{F}\right) \cong \mathrm{GL}_{n}\left(\mathbb{A}_{E}\right)$ in $U_{2 n}$. Then the Eisenstein series $E(f, s)$ on $U_{2 n}\left(\mathbb{A}_{F}\right)$, constructed as in Section 2.B from functions $f$ in the representation space $W_{\sigma}$ on which induced representations $I(s, \sigma)$ are realized for all $s$, is

(1) holomorphic for $\operatorname{Re}(s) \geq 0$, if $\sigma$ is not Galois self-dual,

(2) holomorphic for $\operatorname{Re}(s) \geq 0$, except for a possible simple pole at $s=\frac{1}{2}$, if $\sigma$ is Galois self-dual.

Proof. The Eisenstein series is holomorphic on the imaginary axis $\operatorname{Re}(s)=0$ (see [Mœglin and Waldspurger 1995, Section IV.1.11]). Hence, we may assume $\operatorname{Re}(s)>0$. Suppose that the Eisenstein series $E(f, s)$ on $U_{2 n}\left(\mathbb{A}_{F}\right)$ has a pole at $s=s_{0}>0$ for some $f \in W_{\sigma}$ in the notation of Section 2. Since $s_{0}>0$, the residues at $s=s_{0}$ of $E(f, s)$ when $f \in W_{\sigma}$ span a residual automorphic representation of $U_{2 n}\left(\mathbb{A}_{F}\right)$. But this residual representation is a constituent of the induced representation

$$
\operatorname{Ind}_{P\left(\mathbb{A}_{F}\right)}^{U_{2 n}\left(A_{F}\right)}\left(\sigma \otimes|\operatorname{det}|_{E}^{s_{0}}\right) .
$$

By Proposition 3.5, its Arthur parameter is

$$
\psi=\sigma \otimes v(2),
$$

where $\sigma$ is Galois self-dual and $s_{0}=\frac{1}{2}$. Therefore, the Eisenstein series $E(f, s)$ is holomorphic for $\operatorname{Re}(s)>0$, except for a possible pole at $s=\frac{1}{2}$ if $\sigma$ is Galois selfdual, as claimed. The possible pole is simple, by the general theory of Eisenstein series [Mœglin and Waldspurger 1995, Section IV.1.11]

Remark 4.2. A significant part of Theorem 4.1 can be proved in a different way, without using Mok's work on the Arthur classification for unitary groups [Mok 2015], which is based on the trace formula, and still depends on the stabilization of the twisted trace formula for $\mathrm{GL}_{n}$. 
For instance, if $\sigma$ is not Galois self-dual, the following general argument provides holomorphy of the Eisenstein series for $\operatorname{Re}(s)>0$. By [Harish-Chandra 1968], see also [Mœglin and Waldspurger 1995, Section IV.3.12], a necessary condition for the Eisenstein series $E(f, s)$ to have a pole for $\operatorname{Re}(s)>0$ and some $f \in W_{\sigma}$ is that $\sigma^{w_{0}} \cong \sigma$. But in our case, $\sigma^{w_{0}}=\tilde{\sigma}^{\theta}$, so that $E(f, s)$ is holomorphic for $\operatorname{Re}(s)>0$ and all $f \in W_{\sigma}$ if $\sigma$ is not Galois self-dual.

If $\sigma$ is Galois self-dual, there is a unitarity argument, which gives the analytic behavior of the Eisenstein series for $\operatorname{Re}(s) \geq \frac{1}{2}$. However, the critical strip $0<$ $\operatorname{Re}(s)<\frac{1}{2}$ remains out of reach. For completeness, we include this argument in Section 4.C below.

4.B. Analytic properties of Asai L-functions. The following theorem describes completely the analytic properties of the Asai $L$-functions attached to a cuspidal automorphic representation $\sigma$ of $\mathrm{GL}_{n}\left(\mathbb{A}_{E}\right)$. It is the main result of the paper.

Theorem 4.3. Let $\sigma$ be a cuspidal automorphic representation of $\mathrm{GL}_{n}\left(\mathbb{A}_{E}\right)$. Let $L\left(s, \sigma, r_{A}\right)$ (respectively, $\left.L\left(s, \sigma \otimes \hat{\delta}, r_{A}\right)\right)$ be the Asai (respectively, twisted Asai) $L$-function attached to $\sigma$, where $\hat{\delta}$ is any extension to $\mathbb{A}_{E}^{\times} / E^{\times}$of the quadratic character of $\mathbb{A}_{F}^{\times} / F^{\times}$attached to the extension $E / F$ by class field theory.

(1) If $\sigma$ is not Galois self-dual, that is, $\sigma \neq \tilde{\sigma}^{\theta}$, then $L\left(s, \sigma, r_{A}\right)$ is entire. It is nonzero for $\operatorname{Re}(s) \geq 1$ and $\operatorname{Re}(s) \leq 0$.

(2) If $\sigma$ is Galois self-dual, that is, $\sigma \cong \tilde{\sigma}^{\theta}$, then

(a) $L\left(s, \sigma, r_{A}\right)$ is entire, except for possible simple poles at $s=0$ and $s=1$, and nonzero for $\operatorname{Re}(s) \geq 1$ and $\operatorname{Re}(s) \leq 0$;

(b) exactly one of the L-functions $L\left(s, \sigma, r_{A}\right)$ and $L\left(s, \sigma \otimes \hat{\delta}, r_{A}\right)$ has simple poles at $s=0$ and $s=1$, while the other is holomorphic at those points.

Proof. The idea of the proof goes back to [Shahidi 1981; 1988]. The proof of holomorphy is based on Theorem 2.1, which relates the poles of Eisenstein series to the Asai $L$-functions, and Theorem 4.1 providing the analytic behavior of the Eisenstein series. The nonvanishing, on the other hand, follows from considering the nonconstant term of the Eisenstein series as in [Shahidi 1981] (see also [Shahidi 2010, Section 7]), and using Theorem 4.1 again. It is sufficient to prove the claims for $\operatorname{Re}(s) \geq \frac{1}{2}$, due to the functional equation for Asai $L$-functions.

We begin with the proof of holomorphy. Consider first the case of $\sigma$ not Galois self-dual. According to Theorem 4.1, the Eisenstein series attached to $\sigma$ is holomorphic for $\operatorname{Re}(s)>0$. Assume that $L\left(s, \sigma, r_{A}\right)$ has a pole for $s=s_{0}>0$. Since the poles of $E(f, s)$ for $\operatorname{Re}(s)>0$ coincide, according to Theorem 2.1, with the poles of the ratio

$$
\frac{L\left(2 s, \sigma, r_{A}\right)}{L\left(1+2 s, \sigma, r_{A}\right)},
$$


the pole of the numerator at $2 s=s_{0}>0$ should be canceled by a pole in the denominator. Thus, $L\left(z, \sigma, r_{A}\right)$ should have a pole at $z=s_{0}+1$. Repeating this argument, we obtain a sequence of poles of the Asai $L$-function of the form $s_{0}+M$, where $M$ is any nonnegative integer. This is a contradiction, because $L\left(s, \sigma, r_{A}\right)$ is holomorphic in the right half-plane of absolute convergence of the defining product. Thus, we proved that $L\left(s, \sigma, r_{A}\right)$ is entire.

Consider now the case of $\sigma$ Galois self-dual. By Theorem 4.1, the Eisenstein series $E(f, s)$ attached to $\sigma$ is holomorphic for $\operatorname{Re}(s)>0$, except for a possible simple pole at $s=\frac{1}{2}$. The same argument as in the previous case implies that $L\left(z, \sigma, r_{A}\right)$ is holomorphic for $\operatorname{Re}(z)>0$, except for $z=1$ if the Eisenstein series has a pole at $s=\frac{1}{2}$.

To prove that a possible pole of $L\left(z, \sigma, r_{A}\right)$ at $z=1$ is at most simple, we again apply a similar argument. Suppose $E(f, s)$ has a pole at $s=\frac{1}{2}$. It is simple by Theorem 4.1. If $L\left(z, \sigma, r_{A}\right)$ had a higher order pole at $z=2 s=1$, then Theorem 2.1 would imply that there is a pole in the denominator of the ratio of Asai $L$-functions in $(*)$. But this would mean that the Asai $L$-function has a pole at $z+1=2$. The Eisenstein series is holomorphic at $s=1$, so that the same argument as before gives a sequence of poles at all positive integers, which is a contradiction.

For nonvanishing, consider the nonconstant term $E(f, s)_{\psi}$ of the Eisenstein series $E(f, s)$ with respect to a fixed nontrivial additive character $\psi$ of $F \backslash \mathbb{A}_{F}$. According to [Shahidi 2010, Theorem 7.1.2], we have

$$
E(f, s)_{\psi}(e)=\frac{1}{L^{S}\left(1+2 s, \sigma, r_{A}\right)} \cdot \prod_{v \in S} W_{v}\left(e_{v}\right)
$$

where $e$ and $e_{v}$ are the identity matrices, $W_{v}$ is the $\psi_{v}$-Whittaker function attached to $f$ via a Jacquet integral, $S$ is a finite set of places, containing all archimedean places, outside which $U_{2 n}\left(F_{v}\right), \sigma_{v}$ and $\psi_{v}$ are all unramified, and $L^{S}\left(z, \sigma, r_{A}\right)$ is the partial Asai $L$-function attached to $\sigma$. As in [Shahidi 2010, Section 7.2], there is a choice of $f \in W_{\sigma}$ such that $W_{v}\left(e_{v}\right) \neq 0$ for all $v \in S$. Thus, every zero of $L^{S}\left(1+2 s, \sigma, r_{A}\right)$ for $\operatorname{Re}(s) \geq 0$, equivalently $\operatorname{Re}(1+2 s) \geq 1$, would give a pole of the nonconstant term $E(f, s)_{\psi}$. However, by Theorem 4.1, the Eisenstein series $E(f, s)$, and thus $E(f, s)_{\psi}$ as well, is holomorphic for $\operatorname{Re}(s) \geq 0$, except for a possible pole at $s=\frac{1}{2}$, which may occur only if $\sigma$ is Galois self-dual. Hence, $L^{S}\left(z, \sigma, r_{A}\right)$ has no zeroes for $\operatorname{Re}(z) \geq 1$, except possibly for $z=1$. Since the local $L$-functions are nonvanishing, the same holds for the complete Asai $L$-function $L\left(z, \sigma, r_{A}\right)$.

For $\sigma$ Galois self-dual, the nonvanishing of $L\left(z, \sigma, r_{A}\right)$ at the remaining point $z=1$ follows from the identity

$$
L\left(s, \sigma \times \sigma^{\theta}\right)=L\left(s, \sigma, r_{A}\right) L\left(s, \sigma, r_{A} \otimes \delta_{E / F}\right),
$$


where $L\left(s, \sigma \times \sigma^{\theta}\right)$ is the Rankin-Selberg $L$-function, and recall that the twisted Asai $L$-function equals

$$
L\left(s, \sigma, r_{A} \otimes \delta_{E / F}\right)=L\left(s, \sigma \otimes \hat{\delta}, r_{A}\right)
$$

See [Goldberg 1994] for these identities. The poles of the Rankin-Selberg $L$ function $L\left(s, \sigma \times \sigma^{\theta}\right)$ are known from [Jacquet and Shalika 1981]. For $\sigma$ Galois self-dual it has a simple pole at $s=1$. Since $\sigma \otimes \hat{\delta}$ is Galois self-dual as well, we already proved that both Asai $L$-functions on the right-hand side of $(* *)$ have at most a simple pole at $s=1$. Hence, they are both nonzero at $s=1$, and exactly one of them has a simple pole at $s=1$, as claimed.

Remark 4.4. Once the holomorphy of the Asai and twisted Asai $L$-function is known at some $s_{0}$ with $\operatorname{Re}\left(s_{0}\right)>0$, the argument using the Rankin-Selberg $L$ function at the end of this proof can be applied directly to obtain nonvanishing. However, the result of Jacquet and Shalika [1981] providing analytic properties of the Rankin-Selberg $L$-functions is very deep, and we preferred to give an argument using nonconstant term of the Eisenstein series whenever possible.

4.C. Holomorphy of Eisenstein series using a unitarity argument. We now give a different proof that the Eisenstein $E(f, s)$, attached to a Galois self-dual cuspidal automorphic representation $\sigma$ of $\mathrm{GL}_{n}\left(\mathbb{A}_{F}\right)$ as above, is holomorphic for $\operatorname{Re} \geq \frac{1}{2}$, except for a possible simple pole at $s=\frac{1}{2}$.

It is sufficient to prove that $E(f, s)$ is holomorphic for $\operatorname{Re}(s)>\frac{1}{2}$. Indeed, since we always normalize $\sigma$ to be trivial on $A_{P}\left(F_{\infty}\right)^{\circ}$, the poles of the Eisenstein series are real. Hence, the only possible pole for $\operatorname{Re}(s)=\frac{1}{2}$ is at $s=\frac{1}{2}$. It is at most simple pole, because all poles of Eisenstein series inside the closure of the positive Weyl chamber are without multiplicity [Mœglin and Waldspurger 1995, Section IV.1.11].

Suppose that there is a simple pole of $E(f, s)$ at $s=s_{0}>\frac{1}{2}$. We follow an idea of Kim [2000] based on the fact that residual representations are unitary. The space of residues of $E(f, s)$ at $s=s_{0}$ is a residual representation of $U_{2 n}\left(\mathbb{A}_{F}\right)$, which is a constituent of the induced representation

$$
I\left(s_{0}, \sigma\right)=\operatorname{Ind}_{P\left(\mathbb{A}_{F}\right)}^{U_{2 n}\left(\mathbb{A}_{F}\right)}\left(\sigma|\operatorname{det}|_{E}^{s_{0}}\right) .
$$

In particular, this residual representation is unitary, so that the induced representation should have a unitary constituent. But then the local induced representation at every place $v$ should have a unitary subquotient. Let $v$ be a split nonarchimedean place of $F$ such that $\sigma_{v}$ is unramified. The local induced representation at $v$ is isomorphic to

$$
I\left(s_{0}, \sigma_{v}\right) \cong \operatorname{Ind}_{P\left(F_{v}\right)}^{\mathrm{GL}_{2 n}\left(F_{v}\right)}\left(\sigma_{w_{1}}|\operatorname{det}|_{F_{v}}^{s_{0}} \otimes \tilde{\sigma}_{w_{2}}|\operatorname{det}|_{F_{v}}^{-s_{0}}\right),
$$


where $w_{1}$ and $w_{2}$ are the two places of $E$ lying above $v$. Since $\sigma_{w_{1}}$ and $\sigma_{w_{2}}$ are unramified unitary generic representations of $\mathrm{GL}_{n}\left(F_{v}\right)$, according to [Tadić 1986], they are fully induced representations of the form

$$
\begin{aligned}
& \sigma_{w_{1}} \cong \\
& \quad \operatorname{Ind}_{B_{n}\left(F_{v}\right)}^{\mathrm{GL}_{n}\left(F_{v}\right)}\left(\mu_{1}||^{\alpha_{1}} \otimes \cdots \otimes \mu_{k}||^{\alpha_{k}} \otimes \chi_{1} \otimes \cdots \otimes \chi_{l} \otimes \mu_{k}||^{-\alpha_{k}} \otimes \cdots \otimes \mu_{1}||^{-\alpha_{1}}\right)
\end{aligned}
$$

and

$\tilde{\sigma}_{w_{2}} \cong$

$\operatorname{Ind}_{B_{n}\left(F_{v}\right)}^{\mathrm{GL}_{n}\left(F_{v}\right)}\left(\mu_{1}^{\prime}||^{\beta_{1}} \otimes \cdots \otimes \mu_{k^{\prime}}^{\prime}||^{\beta_{k^{\prime}}} \otimes \chi_{1}^{\prime} \otimes \cdots \otimes \chi_{l^{\prime}}^{\prime} \otimes \mu_{k^{\prime}}^{\prime}||^{-\beta_{k^{\prime}}} \otimes \cdots \otimes \mu_{1}^{\prime}||^{-\beta_{1}}\right)$,

where $B_{n}$ is a Borel subgroup of $\mathrm{GL}_{n}$, the exponents satisfy $0<\alpha_{k}<\cdots<\alpha_{1}<\frac{1}{2}$ and $0<\beta_{k^{\prime}}<\cdots<\beta_{1}<\frac{1}{2}$, and $\mu_{i}, \mu_{i}^{\prime}, \chi_{j}, \chi_{j}^{\prime}$ are unramified unitary characters of $F_{v}^{\times}$. Hence,

$$
\begin{aligned}
I\left(s_{0}, \sigma_{v}\right) \cong \operatorname{Ind}_{B_{2 n}\left(F_{v}\right)}^{\mathrm{GL}_{2 n}\left(F_{v}\right)}\left(\mu_{1}||^{s_{0}+\alpha_{1}} \otimes \cdots \otimes \mu_{k}||^{s_{0}+\alpha_{k}} \otimes \chi_{1}||^{s_{0}} \otimes \cdots \otimes \chi_{l}||^{s_{0}}\right. \\
\otimes \mu_{k}||^{s_{0}-\alpha_{k}} \otimes \cdots \otimes \mu_{1}||^{s_{0}-\alpha_{1}} \\
\otimes \mu_{1}^{\prime}||^{-s_{0}+\beta_{1}} \otimes \cdots \otimes \mu_{k^{\prime}}^{\prime}||^{-s_{0}+\beta_{k^{\prime}}} \otimes \chi_{1}^{\prime}||^{-s_{0}} \otimes \cdots \\
\left.\otimes \chi_{l^{\prime}}^{\prime}||^{-s_{0}} \otimes \mu_{k^{\prime}}^{\prime}||^{-s_{0}-\beta_{k^{\prime}}} \otimes \cdots \otimes \mu_{1}^{\prime}||^{-s_{0}-\beta_{1}}\right) .
\end{aligned}
$$

According to the description of the unitary dual of $\mathrm{GL}_{2 n}\left(F_{v}\right)$ [Tadic 1986], this representation would have a unitary subquotient, only if all the exponents whose absolute value is not smaller than $\frac{1}{2}$, induced with another character to a representation of $\mathrm{GL}_{2}\left(F_{v}\right)$, give a reducible representation with a unitary quotient of Speh type. However, this is possible only if for every such exponent that is not less than $\frac{1}{2}$ in absolute value, there is another exponent such that their difference is exactly 1 .

Having this in mind, consider the largest exponent in the above induced representation. We write this exponent as $s_{0}+\alpha_{1}$, and allow the possibility $\alpha_{1}=0$, which happens in the case $k=0$ as there are no $\alpha_{i}$ 's. There should be another exponent of the form $-s_{0} \pm \beta$, where $\beta=\beta_{j}$ for some $j$ or $\beta=0$, such that

$$
\left(s_{0}+\alpha_{1}\right)-\left(-s_{0} \pm \beta\right)=1 .
$$

But this implies

$$
2 s_{0}+\alpha_{1} \mp \beta=1,
$$

which is possible for $s_{0}>\frac{1}{2}$ only if the sign of $\beta$ is minus and $\alpha_{1}<\beta$. As $\beta$ is certainly not greater than the largest of $\beta_{j}$ 's, it follows that necessarily $\alpha_{1}<\beta_{1}$. However, considering the smallest exponent in the induced representation, that is, $-s_{0}-\beta_{1}$, where again $\beta_{1}$ is set to zero if $l=0$, we obtain the opposite inequality, $\beta_{1}<\alpha_{1}$. This is a contradiction, proving that $I\left(s_{0}, \sigma_{v}\right)$ has not a unitary subquotient 
for $s_{0}>\frac{1}{2}$, and therefore, the Eisenstein series $E(f, s)$ has no pole for $\operatorname{Re}(s)>\frac{1}{2}$, as claimed.

\section{Acknowledgements}

This paper was finalized during our visit to the Erwin Schrödinger Institute in Vienna for the workshop Advances in the Theory of Automorphic Forms and their L-Functions. We are grateful for the invitation and the ever stimulating atmosphere of the Erwin Schrödinger Institute. The first named author would like to thank Goran Muić for some useful discussions.

\section{References}

[Anandavardhanan and Rajan 2005] U. K. Anandavardhanan and C. S. Rajan, "Distinguished representations, base change, and reducibility for unitary groups", Int. Math. Res. Not. 2005:14 (2005), 841-854. MR 2006g:22013 Zbl 1070.22011

[Arthur 2004] J. Arthur, "Automorphic representations of GSp(4)", pp. 65-81 in Contributions to automorphic forms, geometry, and number theory (Baltimore, MD, 2002), edited by H. Hida et al., Johns Hopkins University Press, Baltimore, MD, 2004. MR 2005d:11074 Zbl 1080.11037

[Arthur 2005] J. Arthur, "An introduction to the trace formula", pp. 1-263 in Harmonic analysis, the trace formula, and Shimura varieties, edited by J. Arthur et al., Clay Math. Proc. 4, Amer. Math. Soc., Providence, RI, 2005. MR 2007d:11058 Zbl 1152.11021

[Arthur 2013] J. Arthur, The endoscopic classification of representations: orthogonal and symplectic groups, Amer. Math. Soc. Colloq. Publ. 61, Amer. Math. Soc., Providence, RI, 2013. MR 3135650 Zbl 06231010

[Asai 1977] T. Asai, “On certain Dirichlet series associated with Hilbert modular forms and Rankin's method”, Math. Ann. 226:1 (1977), 81-94. MR 55 \#2761 Zbl 0326.10024

[Belt 2012] D. D. Belt, On the holomorphy of exterior-square L-functions, thesis, Purdue University, West Lafayette, IN, 2012, available at http://search.proquest.com/docview/1237150822. MR 3103748

[Bourbaki 1968] N. Bourbaki, Éléments de mathématique, Fasc. XXXIV: Groupes et algèbres de Lie, Chapitre IV: Groupes de Coxeter et systèmes de Tits. Chapitre V: Groupes engendrés par des réflexions. Chapitre VI: Systèmes de racines, Actualités Scientifiques et Industrielles 1337, Hermann, Paris, 1968. Translated as Lie groups and Lie algebras: chapters 4-6, Springer, Berlin, 2002. MR 39 \#1590 Zbl 0186.33001

[Bump and Friedberg 1990] D. Bump and S. Friedberg, "The exterior square automorphic $L$-functions on GL(n)", pp. 47-65 in Festschrift in honor of I. I. Piatetski-Shapiro on the occasion of his sixtieth birthday, II: Papers in analysis, number theory and automorphic L-functions (Tel Aviv, 1989), edited by S. Gelbart et al., Israel Math. Conf. Proc. 3, Weizmann, Jerusalem, 1990. MR 93d:11050 Zbl 0712.11030

[Bump and Ginzburg 1992] D. Bump and D. Ginzburg, "Symmetric square $L$-functions on GL( $r)$ ", Ann. of Math. (2) 136:1 (1992), 137-205. MR 93i:11058 Zbl 0753.11021

[Casselman and Shahidi 1998] W. Casselman and F. Shahidi, "On irreducibility of standard modules for generic representations", Ann. Sci. École Norm. Sup. (4) 31:4 (1998), 561-589. MR 99f:22028 Zbl 0947.11022 
[Flicker 1988] Y. Z. Flicker, “Twisted tensors and Euler products”, Bull. Soc. Math. France 116:3 (1988), 295-313. MR 89m:11049 Zbl 0674.10026

[Flicker and Zinoviev 1995] Y. Z. Flicker and D. Zinoviev, "On poles of twisted tensor $L$-functions", Proc. Japan Acad. Ser. A Math. Sci. 71:6 (1995), 114-116. MR 96f:11075 Zbl 0983.11023

[Franke and Schwermer 1998] J. Franke and J. Schwermer, "A decomposition of spaces of automorphic forms, and the Eisenstein cohomology of arithmetic groups", Math. Ann. 311:4 (1998), 765-790. MR 99k:11077 Zbl 0924.11042

[Gan et al. 2012] W. T. Gan, B. H. Gross, and D. Prasad, "Symplectic local root numbers, central critical $L$ values, and restriction problems in the representation theory of classical groups", pp. 1-109 in Sur les conjectures de Gross et Prasad, vol. I, Astérisque 346, Société Mathématique de France, Paris, 2012. MR 3202556 Zbl 1280.22019 arXiv 0909.2999

[Godement and Jacquet 1972] R. Godement and H. Jacquet, Zeta functions of simple algebras, Lecture Notes in Math. 260, Springer, Berlin, 1972. MR 49 \#7241 Zbl 0244.12011

[Goldberg 1994] D. Goldberg, "Some results on reducibility for unitary groups and local Asai L-functions", J. Reine Angew. Math. 448 (1994), 65-95. MR 95g:22031 Zbl 0815.11029

[Grbac 2011] N. Grbac, "On the residual spectrum of split classical groups supported in the Siegel maximal parabolic subgroup", Monatsh. Math. 163:3 (2011), 301-314. MR 2012k:11063 Zbl 1247.11080

[Harder et al. 1986] G. Harder, R. P. Langlands, and M. Rapoport, "Algebraische Zyklen auf HilbertBlumenthal-Flächen”, J. Reine Angew. Math. 366 (1986), 53-120. MR 87k:11066 Zbl 0575.14004

[Harish-Chandra 1968] Harish-Chandra, Automorphic forms on semisimple Lie groups, Lecture Notes in Math. 62, Springer, Berlin, 1968. MR 38 \#1216 Zbl 0186.04702

[Jacquet and Shalika 1981] H. Jacquet and J. A. Shalika, "On Euler products and the classification of automorphic forms, II”, Amer. J. Math. 103:4 (1981), 777-815. MR 82m:10050b Zbl 0491.10020

[Jiang et al. 2013] D. Jiang, B. Liu, and L. Zhang, "Poles of certain residual Eisenstein series of classical groups", Pacific J. Math. 264:1 (2013), 83-123. MR 3079762 Zbl 06203663

[Kewat and Raghunathan 2012] P. K. Kewat and R. Raghunathan, "On the local and global exterior square L-functions of GLn”, Math. Res. Lett. 19:4 (2012), 785-804. MR 3008415 Zbl 06165853

[Kim 2000] H. H. Kim, "Langlands-Shahidi method and poles of automorphic $L$-functions, II", Israel J. Math. 117 (2000), 261-284. MR 2001i:11059a Zbl 1041.11035

[Kim and Krishnamurthy 2004] H. H. Kim and M. Krishnamurthy, "Base change lift for odd unitary groups", pp. 116-125 in Functional analysis VIII (Dubrovnik, 2003), edited by D. Bakić et al., Various Publ. Ser. (Aarhus) 47, Aarhus University, Aarhus, 2004. MR 2006a:11154 Zbl 1146.11313

[Kim and Krishnamurthy 2005] H. H. Kim and M. Krishnamurthy, "Stable base change lift from unitary groups to $\mathrm{GL}_{n}$ ”, Int. Math. Res. Pap. 2005:1 (2005), 1-52. MR 2006d:22028 Zbl 1146.11314

[Langlands 1971] R. P. Langlands, Euler products (New Haven, CT, 1967), Yale Mathematical Monographs 1, Yale University Press, New Haven, CT, 1971. MR 54 \#7387 Zbl 0231.20016

[Langlands 1989] R. P. Langlands, "On the classification of irreducible representations of real algebraic groups", pp. 101-170 in Representation theory and harmonic analysis on semisimple Lie groups, edited by P. J. Sally, Jr. and D. A. Vogan, Jr., Math. Surveys Monogr. 31, Amer. Math. Soc., Providence, RI, 1989. MR 91e:22017 Zbl 0741.22009

[Lapid et al. 2004] E. Lapid, G. Muić, and M. Tadić, "On the generic unitary dual of quasisplit classical groups”, Int. Math. Res. Not. 2004:26 (2004), 1335-1354. MR 2005b:22021 Zbl 1079.22015

[Luo et al. 1999] W. Luo, Z. Rudnick, and P. Sarnak, "On the generalized Ramanujan conjecture for GL(n)", pp. 301-310 in Automorphic forms, automorphic representations, and arithmetic (Fort 
Worth, TX, 1996), edited by R. S. Doran et al., Proc. Sympos. Pure Math. 66, Amer. Math. Soc., Providence, RI, 1999. MR 2000e:11072 Zbl 0965.11023

[Mœglin 2008] C. Mœglin, "Formes automorphes de carré intégrable non cuspidales", Manuscripta Math. 127:4 (2008), 411-467. MR 2010i:11071 Zbl 05520091

[Mœglin and Waldspurger 1989] C. Mœglin and J.-L. Waldspurger, "Le spectre résiduel de GL(n)", Ann. Sci. École Norm. Sup. (4) 22:4 (1989), 605-674. MR 91b:22028 Zbl 0696.10023

[Mœglin and Waldspurger 1995] C. Mœglin and J.-L. Waldspurger, Spectral decomposition and Eisenstein series: a paraphrase of the Scriptures, Cambridge Tracts in Mathematics 113, Cambridge University Press, 1995. MR 97d:11083 Zbl 0846.11032

[Mok 2015] C. P. Mok, "Endoscopic classification of representations of quasi-split unitary groups", American Mathematical Society, Providence, RI, 2015. Mem. Amer. Math. Soc.

[Muić 2001] G. Muić, "A proof of Casselman-Shahidi’s conjecture for quasi-split classical groups", Canad. Math. Bull. 44:3 (2001), 298-312. MR 2002f:22015 Zbl 0984.22007

[Rogawski 1990] J. D. Rogawski, Automorphic representations of unitary groups in three variables, Annals of Mathematics Studies 123, Princeton University Press, 1990. MR 91k:22037 Zbl 0724.11031

[Shahidi 1981] F. Shahidi, "On certain L-functions", Amer. J. Math. 103:2 (1981), 297-355. MR 82i: 10030 Zbl 0467.12013

[Shahidi 1985] F. Shahidi, "Local coefficients as Artin factors for real groups", Duke Math. J. 52:4 (1985), 973-1007. MR 87m:11049 Zbl 0674.10027

[Shahidi 1988] F. Shahidi, "On the Ramanujan conjecture and finiteness of poles for certain $L$ functions”, Ann. of Math. (2) 127:3 (1988), 547-584. MR 89h:11021 Zbl 0654.10029

[Shahidi 1990] F. Shahidi, "A proof of Langlands' conjecture on Plancherel measures; complementary series for p-adic groups”, Ann. of Math. (2) 132:2 (1990), 273-330. MR 91m:11095 Zbl 0780.22005

[Shahidi 1992] F. Shahidi, "Twisted endoscopy and reducibility of induced representations for $p$-adic groups”, Duke Math. J. 66:1 (1992), 1-41. MR 93b:22034 Zbl 0785.22022

[Shahidi 2010] F. Shahidi, Eisenstein series and automorphic L-functions, Amer. Math. Soc. Colloq. Publ. 58, Amer. Math. Soc., Providence, RI, 2010. MR 2012d:11119 Zbl 1215.11054

[Speh 1981] B. Speh, "The unitary dual of Gl(3, R) and Gl(4, R)", Math. Ann. 258:2 (1981), 113-133. MR 83i:22025 Zbl 0483.22005

[Tadić 1986] M. Tadić, "Classification of unitary representations in irreducible representations of general linear group (non-Archimedean case)”, Ann. Sci. École Norm. Sup. (4) 19:3 (1986), 335-382. MR 88b:22021 Zbl 0614.22005

[Takeda 2013] S. Takeda, "On a certain metaplectic Eisenstein series and the twisted symmetric square $l$-function", preprint, University of Missouri, Columbia, MO, 2013, available at http:// www.math.missouri.edu/ takedas/analytic_Eisen.pdf.

[Takeda 2014a] S. Takeda, "Metaplectic tensor products for automorphic representations of $\tilde{G L}(r)$ ", preprint, 2014. arXiv 1303.2785

[Takeda 2014b] S. Takeda, "The twisted symmetric square $L$-function of GL(r)", Duke Math. J. 163:1 (2014), 175-266. MR 3161314 Zbl 06279927

[Vogan 1978] D. A. Vogan, Jr., “Gel'fand-Kirillov dimension for Harish-Chandra modules", Invent. Math. 48:1 (1978), 75-98. MR 58 \#22205 Zbl 0389.17002

[Vogan 1986] D. A. Vogan, Jr., “The unitary dual of GL(n) over an Archimedean field”, Invent. Math. 83:3 (1986), 449-505. MR 87i:22042 Zbl 0598.22008 
[Waldspurger 2014a] J.-L. Waldspurger, "Stabilisation de la formule des traces tordue, I: endoscopie tordue sur un corps local", preprint, Institut de Mathématiques de Jussieu, Paris, 2014, available at http://webusers.imj-prg.fr/ jean-loup.waldspurger/stabilisationIfinal.pdf.

[Waldspurger 2014b] J.-L. Waldspurger, "Stabilisation de la formule des traces tordue, II: intégrales orbitales et endoscopie sur un corps local non-archimédien; définitions et énoncés des résultats", preprint, Paris, 2014, available at http://webusers.imj-prg.fr/ jean-loup.waldspurger/ stabilisationIIfinal.pdf.

[Waldspurger 2014c] J.-L. Waldspurger, "Stabilisation de la formule des traces tordue, III: intégrales orbitales et endoscopie sur un corps local non-archimédien; réductions et preuves", preprint, Paris, 2014, available at http://webusers.imj-prg.fr/ jean-loup.waldspurger/stabilisationIIIfinal.pdf.

[Wallach 1979] N. R. Wallach, "Representations of reductive Lie groups", pp. 71-86 in Automorphic forms, representations and L-functions, 1 (Corvallis, OR, 1977), edited by A. Borel and W. Casselman, Proc. Sympos. Pure Math. 33, Amer. Math. Soc., Providence, RI, 1979. MR 80m:22024 Zbl 0421.22006

[Zelevinsky 1980] A. V. Zelevinsky, "Induced representations of reductive p-adic groups, II: On irreducible representations of GL(n)", Ann. Sci. École Norm. Sup. (4) 13:2 (1980), 165-210. MR 83g:22012 Zbl 0441.22014

[Zhang 1997] Y. Zhang, "The holomorphy and nonvanishing of normalized local intertwining operators”, Pacific J. Math. 180:2 (1997), 385-398. MR 98k:22076 Zbl 1073.22502

Received February 17, 2014. Revised April 17, 2014.

NEVEN GRBAC

DEPARTMENT OF MATHEMATICS

UNIVERSITY OF RIJEKA

RADMILE MATEJCIC 2

HR-51000 RIJEKA

CROATIA

neven.grbac@math.uniri.hr

FREYDOON SHAHIDI

Department of Mathematics

PURDUE UNIVERSITY

150 N UNIVERSITY STREET

WEST LAFAYETTE, IN 47907-2067

UNITED STATES

shahidi@math.purdue.edu 


\title{
PACIFIC JOURNAL OF MATHEMATICS
}

\author{
msp.org/pjm
}

Founded in 1951 by E. F. Beckenbach (1906-1982) and F. Wolf (1904-1989)

\section{EDITORS}

Don Blasius (Managing Editor)

Department of Mathematics

University of California

Los Angeles, CA 90095-1555

blasius@math.ucla.edu

\author{
Paul Balmer \\ Department of Mathematics \\ University of California \\ Los Angeles, CA 90095-1555 \\ balmer@math.ucla.edu \\ Robert Finn \\ Department of Mathematics \\ Stanford University \\ Stanford, CA 94305-2125 \\ finn@math.stanford.edu \\ Sorin Popa \\ Department of Mathematics \\ University of California \\ Los Angeles, CA 90095-1555 \\ popa@math.ucla.edu
}

\author{
Vyjayanthi Chari \\ Department of Mathematics \\ University of California \\ Riverside, CA 92521-0135 \\ chari@math.ucr.edu \\ Kefeng Liu \\ Department of Mathematics \\ University of California \\ Los Angeles, CA 90095-1555 \\ liu@math.ucla.edu \\ Jie Qing \\ Department of Mathematics \\ University of California \\ Santa Cruz, CA 95064 \\ qing@ cats.ucsc.edu
}

\section{PRODUCTION}

Silvio Levy, Scientific Editor, production@msp.org

\section{SUPPORTING INSTITUTIONS}

ACADEMIA SINICA, TAIPEI

CALIFORNIA INST. OF TECHNOLOGY

INST. DE MATEMÁTICA PURA E APLICADA

KEIO UNIVERSITY

MATH. SCIENCES RESEARCH INSTITUTE

NEW MEXICO STATE UNIV.

OREGON STATE UNIV.

\author{
STANFORD UNIVERSITY \\ UNIV. OF BRITISH COLUMBIA \\ UNIV. OF CALIFORNIA, BERKELEY \\ UNIV. OF CALIFORNIA, DAVIS \\ UNIV. OF CALIFORNIA, LOS ANGELES \\ UNIV. OF CALIFORNIA, RIVERSIDE \\ UNIV. OF CALIFORNIA, SAN DIEGO \\ UNIV. OF CALIF., SANTA BARBARA
}

\author{
Daryl Cooper \\ Department of Mathematics \\ University of California \\ Santa Barbara, CA 93106-3080 \\ cooper@math.ucsb.edu \\ Jiang-Hua Lu \\ Department of Mathematics \\ The University of Hong Kong \\ Pokfulam Rd., Hong Kong \\ jhlu@maths.hku.hk \\ Paul Yang \\ Department of Mathematics \\ Princeton University \\ Princeton NJ 08544-1000 \\ yang@math.princeton.edu
}

These supporting institutions contribute to the cost of publication of this Journal, but they are not owners or publishers and have no responsibility for its contents or policies.

See inside back cover or msp.org/pjm for submission instructions.

The subscription price for 2015 is US \$420/year for the electronic version, and \$570/year for print and electronic.

Subscriptions, requests for back issues and changes of subscribers address should be sent to Pacific Journal of Mathematics, P.O. Box 4163, Berkeley, CA 94704-0163, U.S.A. The Pacific Journal of Mathematics is indexed by Mathematical Reviews, Zentralblatt MATH, PASCAL CNRS Index, Referativnyi Zhurnal, Current Mathematical Publications and Web of Knowledge (Science Citation Index).

The Pacific Journal of Mathematics (ISSN 0030-8730) at the University of California, c/o Department of Mathematics, 798 Evans Hall \#3840, Berkeley, CA 94720-3840, is published twelve times a year. Periodical rate postage paid at Berkeley, CA 94704, and additional mailing offices. POSTMASTER: send address changes to Pacific Journal of Mathematics, P.O. Box 4163, Berkeley, CA 94704-0163.

PJM peer review and production are managed by EditFLOW ${ }^{\circledR}$ from Mathematical Sciences Publishers.

\section{PUBLISHED BY}

\section{mathematical sciences publishers \\ nonprofit scientific publishing}

http://msp.org/

(C) 2015 Mathematical Sciences Publishers 


\section{PACIFIC JOURNAL OF MATHEMATICS}

Volume $276 \quad$ No. $1 \quad$ July 2015

On the degree of certain local $L$-functions

U. K. ANANDAVARdhanan and AmiYa Kumar MondaL

Torus actions and tensor products of intersection cohomology

ASILATA BAPAT

Cyclicity in Dirichlet-type spaces and extremal polynomials II: functions on the bidisk

Catherine Bénéteau, Alberto A. Condori, Constanze Liaw,

DANIEl SECo and Alan A. SOlA

Compactness results for sequences of approximate biharmonic maps

CHRISTINE BREINER and TOBIAS LAMM

Criteria for vanishing of Tor over complete intersections

Olgur Celikbas, Srikanth B. Iyengar, Greg PiePMeyer and

ROGER WIEGAND

Convex solutions to the power-of-mean curvature flow

\section{SHIBING CHEN}

Constructions of periodic minimal surfaces and minimal annuli in $\mathrm{Sol}_{3}$

CHRISTOPHE DESMONTS

Quasi-exceptional domains

ALEXANDRE EREMENKO and ERIK LUNDBERG

Endoscopic transfer for unitary groups and holomorphy of Asai $L$-functions

NEVEN GRBAC and FREYDOON SHAHIDI

Quasiconformal harmonic mappings between Dini-smooth Jordan domains

DAVID KALAJ

Semisimple super Tannakian categories with a small tensor generator

THOMAS KRÄMER and RAINER WEISSAUER

On maximal Lindenstrauss spaces

PETR PETRÁČEK and JiŘí SPURNÝ 